\title{
Simulation of Gamma-Ray and Neutron Spectrometers for Microsatellite Missions
}

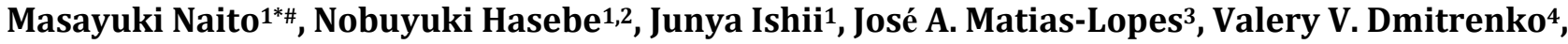 \\ Christian Wöhler ${ }^{5}$, Kyeong Ja Kim ${ }^{6}$
}

\author{
${ }^{1}$ School of Advanced Science and Engineering, Waseda University, Tokyo, Japan \\ ${ }^{2}$ Research Institute for Science and Engineering, Waseda University, Tokyo, Japan \\ ${ }^{3}$ Department of Physics, University of Coimbra, Coimbra, Portugal \\ ${ }^{4}$ National Research Nuclear University MEPhI, Moscow, Russia \\ ${ }^{5}$ Image Analysis Group, Dortmund University of Technology, Dortmund, Germany \\ ${ }^{6}$ Korea Institute of Gescience and Mineral Resources, Daejeon, South Korea \\ Email: *com-nm@a kane.waseda.jp
}

How to cite this paper: Naito, M., Hasebe, N., Ishii, J., Matias-Lopes, J.A., Dmitrenko, V.V., Wöhler, C. and Kim, K.J. (2018) Simulation of Gamma-Ray and Neutron Spectrometers for Microsatellite Missions. Journal of Geoscience and Environment Protection, 6, 84-111.

https://doi.org/10.4236/gep.2018.68009

Received: July 31, 2018

Accepted: August 26, 2018

Published: August 29, 2018

Copyright () 2018 by authors and Scientific Research Publishing Inc. This work is licensed under the Creative Commons Attribution International License (CC BY 4.0).

http://creativecommons.org/licenses/by/4.0/

\begin{abstract}
Microsatellites have recently opened windows of frequent and low cost missions for planetary exploration. The performance of gamma-ray and neutron spectrometers on future microsatellite missions is simulated to assess the possibility of observation of hydrogen and major elements, given their concentration on the observation target. The measured elemental abundance will provide important geological constraints, and some of them may serve as space resources. Four different types of target bodies with various hydrogen concentrations in the range of $0-20,000 \mathrm{ppm}$ are assumed as target compositions; Earth's core, C-type, S-type and Martian meteorites. Gamma-ray and neutron emission rates show unique footprints that are related to the different elemental compositions. The starting point is the solid angle subtended between observation target and spectrometers that allow estimating the gamma-ray and neutron count rates emitted by the celestial bodies. In this work, three types of gamma-ray detectors; high-purity germanium (HPGe), $\mathrm{CeBr}_{3}$ and $\mathrm{LaBr}_{3}(\mathrm{Ce})$, a neutron spectrometer combining a lithium glass scintillator with a boron loaded plastic scintillator and a dual mode spectrometer $\mathrm{Cs}_{2} \mathrm{LiYCl}_{6}(\mathrm{Ce})$ (CLYC) are simulated, focusing on their observation backgrounds as a model case for microsatellite based measurements. The background count level of both gamma-ray (except for the $\mathrm{LaBr}_{3}$ detector) and neutron count rates was negligible under these particular conditions. The gamma-ray detectors were compared by the figure of merit, which was determined by their efficiency and energy resolution. It was found that each detector has unique advantages. The HPGe detector has the highest figure of
\end{abstract}

\#Equal contributor. 
merit due to its excellent energy resolution, whereas the CLYC detector is low in weight and power consumption due to its dual sensitivity to gamma-ray and neutron. The $\mathrm{CeBr}_{3}$ detector is an intermediate choice. The neutron count rates are calculated separately in three energy ranges, i.e., thermal $(<0.5$ $\mathrm{eV})$, epithermal $(0.5 \mathrm{eV}-500 \mathrm{keV})$, and fast $(>500 \mathrm{keV})$, as a function of the hydrogen concentration in the $0-20,000$ ppm range. The thermal and epithermal neutron count rates are found to decrease with hydrogen concentration, while the fast neutron count rate increases with the target average atomic mass. The optimal detector should be decided by the mission restraints on mass, power consumption, and heat thermal design.

\section{Keywords}

Gamma-Ray Spectrometer, Neutron Spectrometer, Microsatellite. High Purity Germanium, $\mathrm{CeBr}_{3}, \mathrm{LaBr}_{3}(\mathrm{Ce}), \mathrm{CLYC}$

\section{Introduction}

There are over 10,000 near Earth asteroids (NEAs). NEAs are rocky celestial bodies in the wide size range of $1 \mathrm{~m}-10 \mathrm{~km}$ and are thought to be the building blocks of planets and/or their satellites. Some of them are primordial material that has never differentiated before. Other asteroids are pieces of planetary bodies that were broken apart by a collision during the phase of planet formation. The exploration of these NEAs is closely associated with the study on how the solar system formed and evolved. They are also attractive for sustainability to maintain human activity. NEAs provide massive storages of valuable resources, including hydrogen and rare Earth elements. Therefore, space exploration for NEAs is quite important from the perspectives of science and space utilization in future missions.

As a result of the recent progress of material and information technology, microsatellites with a miniaturized ion engine have opened a new window for deep space explorations. Those small satellites demonstrating low cost, quick delivery, and high performance have resulted in its increasing demand, and $\sim 50 \mathrm{~kg}$ class microsatellites will be used as the next step of the near future. Using a miniature deep space probe, NEA flyby/rendezvous missions would be very interesting to take in deep space exploration [1] [2] [3] [4]. Such microsatellites have small and lightweight engines and can approach small bodies moving near the orbit of Earth. Moreover, they offer a very fast turnaround and an inexpensive means of exploring well-focused, small scale science objectives while providing unique opportunities for students pursuing master's and doctoral courses, young scientists, and engineers, to gain hands-on experience of satellite and payload engineering. Driven by their own vision and efforts, university teams will be able to launch their own satellite into space, thus reaching a new horizon for space research. 
Remote gamma-ray and neutron flux measurements are powerful and unique nuclear spectrometry tools that allow to measure elemental concentrations and their distributions on the planetary surface and in the subsurface material. Information about elemental abundances on a planetary body, along with its size, mass and orbital information, is essential to understand its formation and evolution. Planetary missions such as Apollo [5], NEAR [6], Lunar Prospector [7], Mars Odyssey [8], SELENE (Kaguya) [9], Chang'E-1 [10] and -2 [11], MESSENGER [12], LRO [13], MSL [14], and Dawn [15] have all employed nuclear spectrometers for the assessment of elemental composition. These missions have achieved remarkable success, leading to essential progress in lunar and planetary sciences.

Nuclear spectroscopy is a very convenient and useful technique for NEA space explorations as it allows for determining the global distribution of elemental abundances by orbital measurement. Microsatellite missions to NEAs will characterize the asteroid geology, shape, and elemental and mineralogical composition, allowing assessing their value as a space resource [3] [4]. On the other hand, microsatellite missions are severely restricted in payload weight and electric power consumption when compared with large scale space explorations.

Gamma-ray spectrometer tends to require longer acquisition times and to be heavier comparing to the other observation methods such as reflectance spectra and X-ray spectroscopy, due to gamma-ray penetrativity (e.g. [6] [16]). High sensitivity, light weight and low electric power consumption are requested for the installation of gamma-ray spectrometer on a microsatellite. In this work, four gamma-ray spectrometers and two neutron spectrometers onboard microsatellites are evaluated for the determination of elemental abundance in planetary surface material by numerical simulation.

There are two fundamental gamma-ray emission processes: through decay of radioactive nuclei and by nuclear interactions between neutrons and target nuclei. Celestial bodies with thin or no atmosphere are always exposed to galactic cosmic ray (GCR) particles which mainly consist of hydrogen and helium nuclei. The GCR particles produce fast neutrons through nuclear reactions with planetary nuclei. Fast neutrons lose energy repeatedly by scattering with the target nuclei in the planetary body until becoming thermal neutrons. Finally, those thermal neutrons are captured by the nuclei. A fraction of the neutrons escapes the planetary surface in the process of energy attenuation. On the other hand, the nuclei excited by inelastic scattering or capture of neutrons emit gamma-rays with unique energies. Since the gamma-ray intensity by these processes depends on the neutron flux, simultaneous measurements of both gamma-ray and neutrons are important.

Neutron spectroscopy is useful not only for gamma-ray count correction but also for determination of the hydrogen concentration and the average atomic mass of planetary surface material (e.g. [17] [18]). In general, elements of large atomic mass produce a large number of fast neutrons by nuclear reactions [19] 
[20], whereas light elements, especially hydrogen, act as an effective moderator [21]. Hence, neutron fluxes are useful to determine the concentration of hydrogen and metallic materials with a large average atomic mass. Spatial distributions of the neutron fluxes may reflect regional geologic features on the planetary surface (e.g. [19] [22]). Moreover, it will be important for future manned exploration to assess the confidence with which the presence of water on the NEAs can be measured.

$\mathrm{X}$-ray fluorescence and ultraviolet, visible and infrared reflectance measurement have also often been employed to infer elemental abundances (e.g. [23] [24] [25]). The Observation depth of these two methods is micro meter order, while that of the nuclear spectroscopy measure is a few tens of centimeters. $\mathrm{X}$-ray fluorescence analysis measures characteristic X-rays induced by solar $\mathrm{X}$-ray excitation. In the cases where the solar X-ray intensity is low such as celestial bodies far away from the Sun, on the night side or in the polar region, measurement becomes very difficult or practically impossible, the Sun being the source of excitation. Spectral reflectance measurements are superior both with respect to space resolution and sensitivity compared to nuclear spectrometers. The reflectance method determines absorption wavelengths of minerals, but it does not allow assessing the concentration of individual elements, being also not applicable where the solar intensity is low as well as the $\mathrm{X}$-ray spectroscopy. On the other hand, the nuclear spectroscopy directly measures the elemental concentration in the surface material of celestial bodies. In this respect, nuclear spectroscopy and reflectance spectroscopy are complementary to one another. Using such datasets in a combined way will allow for creating high resolution elemental maps [26] [27] [28]. Therefore, the development of nuclear spectrometer is of essential importance for planetary exploration.

In the second chapter of this paper, geometric and detectors of our simulations are described with indexes of evaluation. The third chapter shows gamma-ray and neutron emission rates from observation targets of different elemental composition, and gamma-ray and neutron detectors are also compared from the view points of elemental identification. Finally, the selection of detectors for microsatellite missions is discussed.

\section{Simulation Methods}

In the simulation of emission and detection of gamma-rays and neutrons, we divided the calculation into three steps; 1) solid angle $\omega$ subtended by the observation target as seen from the detector, 2) radiation emitted from planetary surface, and 3) radiation detected by nuclear spectrometers. Steps 1), 2), and 3) correspond to calculating the transport from the observation target to the detector, the emission rates of gamma-rays and neutrons, and the detection efficiency including energy resolution, respectively. In this work, the results of steps 2) and 3) were obtained by Monte Carlo simulation.

PHITS (Particle and Heavy Ion Transport code System) [29] was employed as 
the simulation tool. It is described in detail by Sato et al. [29]. PHITS employs the intra-nuclear cascade models JAM [30] and INCL [31] as well as the nuclear and atomic data library JENDL-4.0 [32] for both transport and nuclear interaction. Nucleus transport and interaction are simulated using the quantum molecular dynamics model JQMD [33]. Evaporation and fission by hadron and nucleus reactions are calculated by the GEM model [34]. The combination of these models allows the simulations of particles across large energy ranges. The simulation details are described in the following section.

\subsection{Geometrical Configuration and Projectiles}

\subsubsection{Geometry}

Schematic drawings of the calculation geometries are shown in Figure 1.

The $\omega$ normalized to $4 \pi$ was calculated as

$$
\omega=\frac{1}{2 \pi} \int_{F O V} \frac{\cos \Phi}{l^{2}(\theta)} d S
$$

where $F O V$ is the detector field of view. The parameters in this equation are these given in Figure 1(c). In most of cases, the NEAs have irregular shapes rather than spherical shapes. However, we considered the target as a sphere with equivalent volume here. Since $\cos \Phi$ can be shown as $(L-R \cos \theta) / l$, Equation (1) is calculated as

$$
\omega=\frac{R}{2 L^{2}}\left(\frac{R^{2}-L^{2}}{l_{\max }}+l_{\max }+2 R\right)
$$

The value of $\omega$ has been tested by using the parameter $H / R$ in the range of 0.5 - 5.0 In this range, the observation target is inside the FOV; i.e., $l_{\max }=\sqrt{L^{2}-R^{2}}$. Therefore, we can obtain $\omega=R^{2} /\left(L^{2}\right)$. The relation between $H / R$ and $\omega$ is shown in Figure 2. The values in this figure are normalized to those of $H / R=2.0$. When observation backgrounds are negligible, minimum acquisition time for detection varies depending on reciprocal of $\omega$. The normalized minimum acquisition time depending on $H / R$ is also shown in Figure 2. Including $\omega$, the gamma-ray and neutron emission spectra were implemented into the gamma-ray and neutron detectors, respectively.

The gamma-ray and neutron emissions are obtained based on the nuclear interactions of GCR particles with both the observation target and the spacecraft. The sample volume was assumed to be $20 \mathrm{~m} \times 20 \mathrm{~m} \times 20 \mathrm{~m}$, while gamma-ray and neutron spectra emitted from the central $10 \mathrm{~m} \times 10 \mathrm{~m}$ of this volume surface are obtained. The sample compositions of elements are described in the next section. The spacecraft was assumed as being made of $30-100 \mathrm{~kg}$ aluminum charged with xenon fuel in the $1.0-10 \mathrm{~kg}$ range. The case of no fuel was also considered for the estimation of background at the end of mission. $50 \mathrm{~kg}$ of hydrazine fuel with its oxidizer $\left(\mathrm{H}_{2} \mathrm{~N}_{4}+1.3 \mathrm{~N}_{2} \mathrm{O}_{4}\right)$ was considered in order to compare backgrounds with those originated from electric and chemical engines. The hydrazine mass is decided as delivering the similar specific impulse to that 


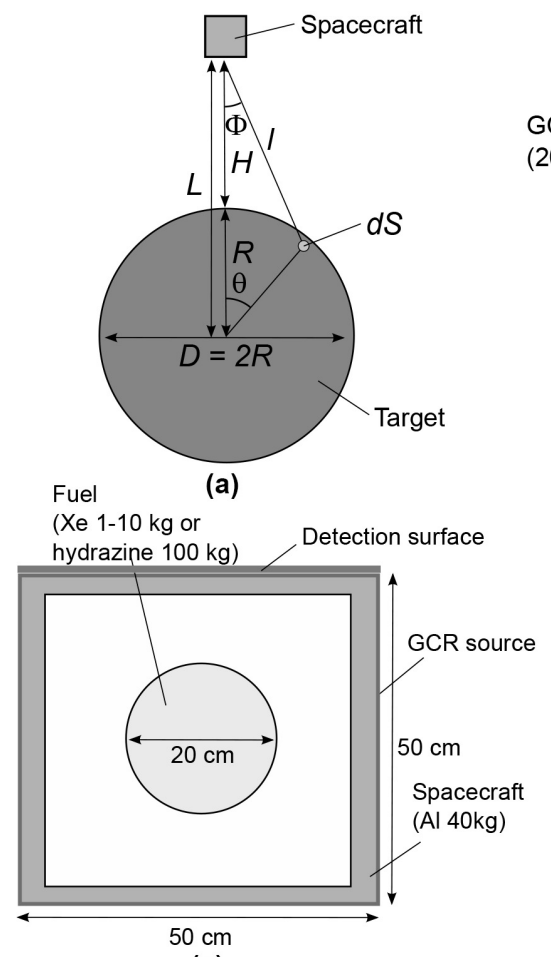

(c)

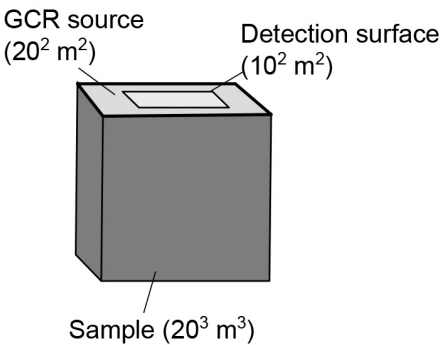

(b)

Gamma-ray/neutron spectrometer

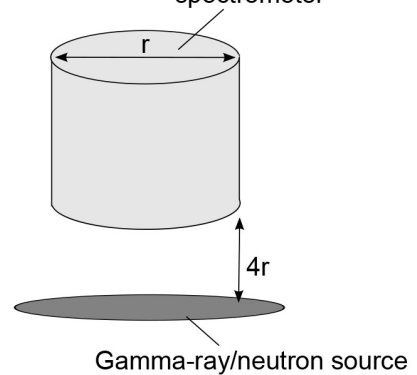

(d)

Figure 1. Schematic drawing of simulation geometry. The calculation was divided into three steps, 1), 2), and 3). (a) and (d) corresponds to steps 1) and 3), respectively. (b) and (c) were used for step 2).

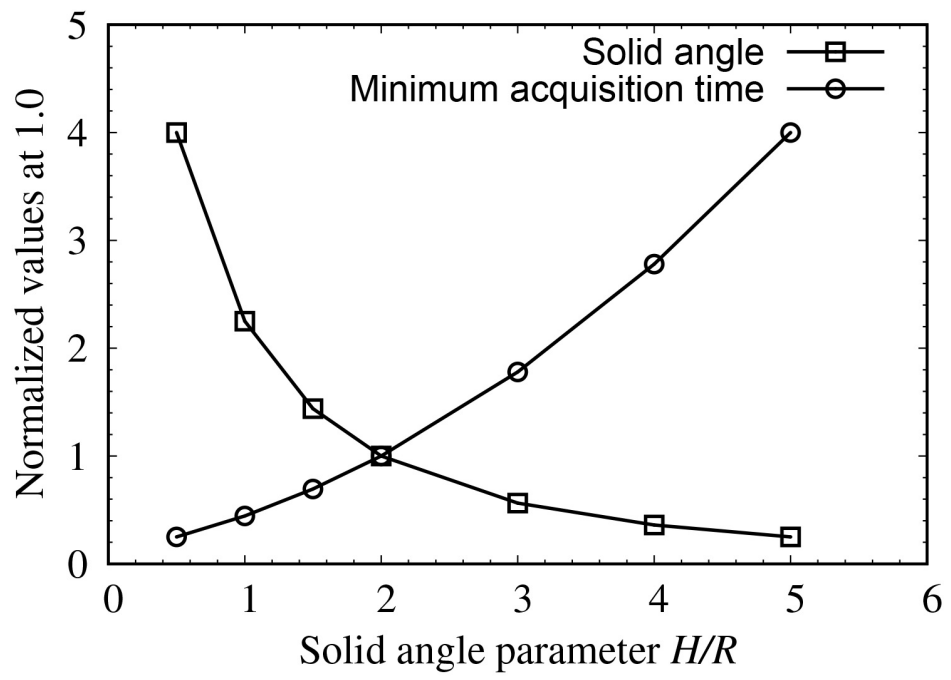

Figure 2. The solid angle dependence on $H / R$. Minimum acquisition time dependence when observation backgrounds are negligible is also shown.

of $5.0 \mathrm{~kg}$ xenon since an ion thruster engine has $\sim 7-10$ times larger specific impulse than chemical propulsion system [35]. The backgrounds emitted by a spacecraft similar to Mars Odyssey (331.8 kg spacecraft with $348.7 \mathrm{~kg}$ fuel [36]) were also estimated for the comparison as a typical case of large scale missions. 


\subsubsection{Target Compositions}

NEAs are classified by their reflectance spectra and orbital parameters [37]. M-type and X-type asteroids are mainly composed by iron and siderophile elements (e.g. [38]). Those together with undifferentiated primitive asteroids are expected to be appealing sources of space resources [39] for microsatellite based exploration. Four types of elemental compositions have been selected to simulate these target asteroids just described.

One is the type of Earth's core with some light elements [40]. This is the profile used for M-type and X-type asteroids since both asteroid types are considered to be produced by collisions that remove crust from the celestial body with a metallic core. The others are the elemental compositions of meteorites which are considered to originate from different types of planetary bodies; C-type and S-type asteroids, and Mars [41] [42] [43]. The C-type asteroid is considered to be the most primitive celestial body with large abundances of hydrogen and carbon [41]. The S-type asteroid is a primitive celestial body next to the C-type, being higher $\mathrm{Si}$ and $\mathrm{Ca}$, and lower $\mathrm{C}$ and $\mathrm{H}$ concentrations than C-type asteroid [42]. The Martian meteorite was selected as a typical basaltic composition of a body finishing thermal evolution [44]. All the considered asteroid type specific elemental compositions are listed with their average atomic mass $\langle A\rangle$ in Table 1. To check the effects of hydrogen concentration, the water equivalent hydrogen concentrations in C-type and Martian compositions were varied in the 0 - 20,000 ppm range.

For the simulations of gamma-rays from radioactive elements $\mathrm{K}, \mathrm{Th}$, and $\mathrm{U}$, characteristic gamma-ray lines from the relevant radioisotope decays of these elements have been added to the gamma-ray emission spectra obtained by the simulations. The potassium concentration was varied in the $0-1000 \mathrm{ppm}$ range while the thorium and uranium concentrations were used as variables in the 0 $1000 \mathrm{ppb}$ range.

\subsubsection{Projectiles}

The GCR Hydrogen and helium particles were chosen as primaries hitting the asteroid surface. Their energy fluxes $J$ were obtained after [45] [46].

$$
\begin{aligned}
& J(E, \phi)=\omega_{g c r} C \times \frac{E\left(E+2 m_{p} c^{2}\right)\left(E+\chi+\phi e \times \frac{Z}{A}\right)^{-\gamma}}{\left(E+\phi e \times \frac{Z}{A}\right)\left(E+2 m_{p} c^{2}+\phi e \times \frac{Z}{A}\right)^{-2}}\left[\mathrm{~cm}^{-2} \cdot \mathrm{s}^{-1}(\mathrm{MeV} / \mathrm{n})^{-1}\right] \\
& \chi=a \exp (-b E)
\end{aligned}
$$

Here, $E(\mathrm{MeV} / \mathrm{n}), \quad \phi(\mathrm{MV}), m_{p} c^{2}(\mathrm{MeV}), Z, A$, and $\gamma$ are particle kinetic energy, solar modulation parameter, proton rest mass energy, atomic number, mass number and power law index, respectively. $C\left(\mathrm{~cm}^{-2} \cdot \mathrm{s}^{-1}(\mathrm{MeV} / \mathrm{n})^{-1}\right), a$ $(\mathrm{MeV})$, and $b\left(\mathrm{MeV}^{-1}\right)$ are normalized constants. The values of constants and $\gamma$ for hydrogen and helium were determined from the PAMELA measurements during 2006-2007 [47] [48]. These values are summarized in Table 2. The value 
Table 1. The elemental compositions (wt\%) of Earth's core, C-type and S-type asteroids, and Martian surface material (soil) assumed for our simulations [40] [41] [42] [43].

\begin{tabular}{ccccc}
\hline & Earth's core & C-type & S-type & Martian \\
\hline $\mathrm{H}$ & 0.60 & 2.02 & 0.331 & ----- \\
$\mathrm{C}$ & ----- & 3.46 & 1.03 & ---- \\
$\mathrm{O}$ & 2.05 & 46.5 & 38.9 & 41.4 \\
$\mathrm{Na}$ & ----- & 0.491 & 0.631 & 0.789 \\
$\mathrm{Mg}$ & ----- & 9.55 & 14.1 & 9.24 \\
$\mathrm{Al}$ & ----- & 0.871 & 1.19 & 3.29 \\
$\mathrm{Si}$ & ----- & 10.7 & 17.6 & 21.7 \\
$\mathrm{~S}$ & ----- & 5.27 & 1.66 & 0.170 \\
$\mathrm{~K}$ & ----- & ---- & 0.08 & 0.08 \\
$\mathrm{Ca}$ & ----- & 0.930 & 1.21 & 5.34 \\
$\mathrm{Ti}$ & ----- & ----- & 0.08 & 0.460 \\
$\mathrm{Fe}$ & 89.6 & 18.6 & 20.7 & 15.0 \\
$\mathrm{Ni}$ & 5.40 & 1.10 & 1.12 & ----- \\
$\mathrm{Others}$ & 0.300 & 0.508 & 1.37 & 2.52 \\
$<A>$ & 40.2 & 15.5 & 21.7 & 23.1 \\
\hline
\end{tabular}

Table 2. Constants used in the calculation for hydrogen and helium GCR fluxes. $\gamma$ defines power law index of energy spectra, and $C$, $a$ and $b$ were normalized factors for the PAMERA observation results [47] [48].

\begin{tabular}{ccccc}
\hline Nuclide & $C$ & $\mathrm{a}$ & $\mathrm{b}$ & $\gamma$ \\
\hline $\mathrm{H}$ & $1.24 \times 10^{6}$ & 780 & $2.50 \times 10^{-4}$ & 2.65 \\
$\mathrm{He}$ & $2.26 \times 10^{5}$ & 660 & $1.40 \times 10^{-4}$ & 2.77 \\
\hline
\end{tabular}

of $\phi$ was fixed to $440 \mathrm{MV}$, which corresponds to the above 2006-2007 measurement periods [49]. The energy spectra obtained by Equation (3) were shown in Figure 3. The data acquired by PAMELA during 2006-2007 and BESS in 1997 are also shown for cross check of Equation (3) [47] [48] [50]. The solar activity during 1997 was a solar minimum activity phase as well as the PAMELA measurement periods [49]. Hence, it is considered that the modulation parameter of $440 \mathrm{MV}$ is consistent with both periods. It is found that Equation (3) reproduces GCR fluxes well, especially hydrogen flux. We used the approximation of the GCR solid angle seen from the flat celestial body surface as $\pi$ i.e., $\omega_{g c r}=1 / 4$. The GCR density at the asteroid surface was fixed to $1.0 \times 10^{4}$ particles $/ \mathrm{m}^{2}$, corresponding to an exposure time of $\sim 1.0 \mathrm{~s}$.

\subsection{Detectors for Gamma-Ray and Neutron}

\subsubsection{Gamma-Ray Detector}

For the evaluation of gamma-ray detector performance, important parameters are its detection efficiency $\varepsilon(E)$ and energy resolution $R(E)$ as a function of 


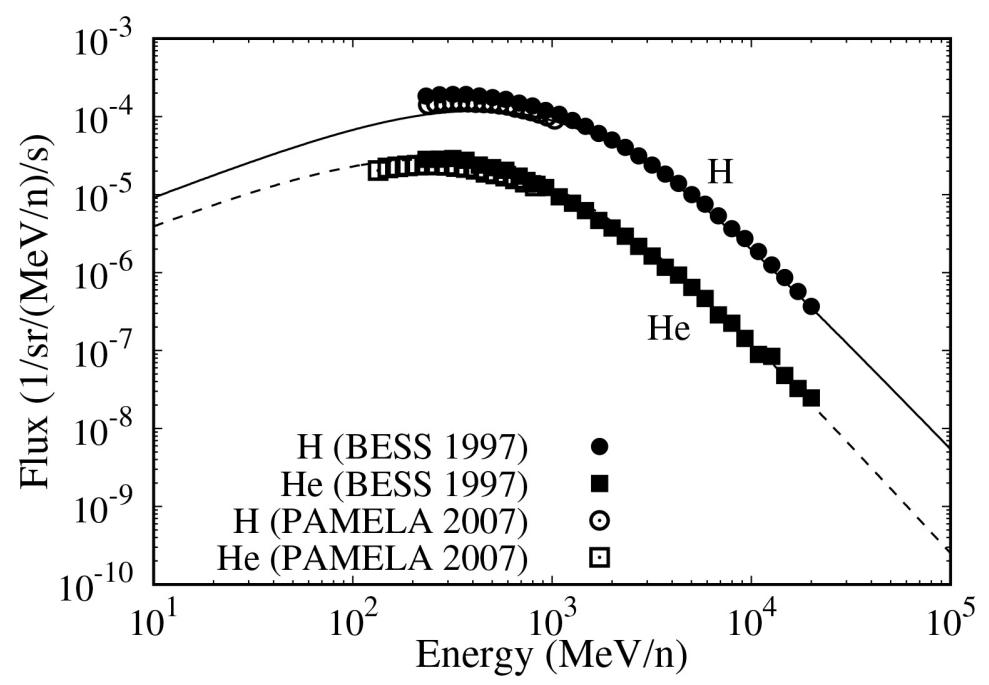

Figure 3. GCR energy spectra. Solid and broken lines represent hydrogen and helium, respectively. The lines show the fluxes obtained by Equation (3) with the parameters in Table 2. Open and close symbols show BESS and PAMELA results, respectively [47] [48] [50].

energy $E$. When the gamma-ray count rates of interest are low, the minimum detectable activity, $D_{\min }$, is given by

$$
\begin{aligned}
D_{\min } & =\frac{N_{\min }}{\varepsilon f t} \\
& =\frac{F_{\text {min }}}{\varepsilon(E) f t}\left(\left[2 n \bar{B}+F_{\text {min }}^{2} / 4\right]^{1 / 2}+F_{\text {min }} / 2\right)
\end{aligned}
$$

where $N_{\min }, f$, and $t$ are the minimum of detectable counts $N$, the decay branching ratio, and acquisition time, respectively [51]. $N_{\min }$ is obtained from the fractional reciprocal error minimum $F_{\min }$, the number of channels defined around the peak $n$ determined by the energy resolution $R(E)$, and the background average $\bar{B}$. The fractional reciprocal error $F$ is called the detector figure of merit and is also used as the parameter for detector performance evaluation. In the case of $N \ll \bar{B}, F$ is given as $N \sqrt{t / 2 n \bar{B}}$. Therefore, the relation between $F, \varepsilon(E), R(E)$, is $F \propto \sqrt{\varepsilon(E) / R(E)}$. As stated above, we use $F$, to compare the gamma-ray detectors performances. A high-purity germanium semiconductor (HPGe), a $\mathrm{CeBr}_{3}$, a $\mathrm{LaBr}_{3}(\mathrm{Ce})$, and $\mathrm{Cs}_{2} \mathrm{LiYCl}_{6}(\mathrm{Ce})$ (CLYC) scintillators are taken as the gamma-ray detectors in order to realize the optimal detection system.

Semiconductors and scintillators have been studied and used as gamma-ray detectors. Generally, scintillators have a large atomic mass and high density, which results in a high detection efficiency, while the energy resolution is low compared to semiconductor detectors. The Ge, CdZnTe, and CdTe detectors are the most commonly applied semiconductor radiation detectors. Semiconductors show a high energy resolution due to their low $\varepsilon$-values $(2.98 \mathrm{eV}$ for $\mathrm{Ge}, 5.0 \mathrm{eV}$ for CdZnTe, and 4.43 for $\mathrm{CdTe}$ ) [52]. On the other hand, density and atomic 
mass of Ge are lower than the scintillator's ones. Compound semiconductors with a high density and large atomic mass such as CdZnTe and CdTe have been studied for their high detection efficiency. However, they show problems in transport properties and limitations in achieving the needed thickness for gamma-ray detection [53]. A lot of devices are required for the large capacity of compound semiconductor detector. We evaluated and compared a semiconductor detector and several scintillators as model cases of gamma-ray detectors. The HPGe, which have been employed in previous missions such as Mars Odyssey [8], SELENE [9], and MESSENGER [12], was selected as the semiconductor detector. $\mathrm{CeBr}_{3}, \mathrm{LaBr}_{3}(\mathrm{Ce})$ and $\mathrm{CLYC}$ were selected as scintillators having the highest energy resolution. Actually, $\mathrm{CeBr}_{3}$ and $\mathrm{LaBr}_{3}(\mathrm{Ce})$ scintillators have been studied and compared as gamma-ray detectors for space missions (e.g. [54] [55]). A $\mathrm{LaBr}_{3}(\mathrm{Ce})$ scintillator have been loaded on Chang'E-2 [11] and will also be used in Bepi Colombo mission [54].

The HPGe achieved energy resolution of $\sim 3.0 \mathrm{keV}$ FWHM at $1332 \mathrm{keV}$ in SELENE ground test [56], requiring cooling below $\sim 100 \mathrm{~K}$ for optimal operation. This value corresponds to $\sim 2.1 \mathrm{keV}$ FWHM at $662 \mathrm{keV}$ by the square root law of energy. Previous missions with the HPGe also employed a refrigerator, increasing the weight and power consumption of the gamma-ray spectrometer as well as imposing a heat flow on the spacecraft. Moreover, the energy resolution degrades by the radiation damage during cruising phase. Although the degradation of the energy resolution can be recovered by crystal annealing, the actual energy resolution in previous missions were worse: $3.9 \mathrm{keV}$ FWHM in Mars Odyssey [8] and 6 - $7 \mathrm{keV}$ FWHM in SELENE [56] at $1461 \mathrm{keV}$, and $4.8 \mathrm{keV}$ FWHM in MESSENGER at $1368 \mathrm{keV}$ [57]. In our calculations, we assumed the highest energy resolution achieved by Mars Odyssey. $\mathrm{The}^{\mathrm{CeBr}} \mathrm{H}_{3}$ scintillator has larger average atomic number than the HPGe detector, so it has a higher detection efficiency for the same depth, while its energy resolution is inferior to that of HPGe, showing $~ 31.1 \mathrm{keV}$ FWHM at $663 \mathrm{keV}$ [55]. $\mathrm{LaBr}_{3}(\mathrm{Ce})$ shows similar detection efficiency to $\mathrm{CeBr}_{3}$ with superior energy resolution $(\sim 25.8 \mathrm{keV}$ FWHM at $663 \mathrm{keV}$ [55]) although it introduces internal background due to ${ }^{138} \mathrm{La}$ radioactive decay. In this study, the gamma-ray flux due to the internal background of $\mathrm{LaBr}_{3}(\mathrm{Ce})$ was also estimated. ${ }^{138} \mathrm{La}$ decays with half-life of $\sim 10^{11}$ years in two decay modes; electron capture (65.5\%) and beta decay (34.5\%) [58]. According to Camp et al. [59], the internal background of $\operatorname{LaBr}_{3}(\mathrm{Ce})$ is $\sim 1.53$ $\mathrm{Bq} / \mathrm{cm}^{3}$. The electron capture reaction emits gamma-rays with $1.44 \mathrm{MeV}$, while the beta decay emits $789 \mathrm{keV}$ gamma-rays with a Q-value of $1.05 \mathrm{MeV}$. Electron energies by the beta decay were assumed to follow a Fermi profile spectrum, extracted from the total measured spectrum by numerical deconvolution, as reported by Quarati et al. [60]. The CLYC detector has recently been studied due to its sensitivity to both gamma-ray and neutron and their discrimination by pulse shape (e.g. [61] [62] [63] [64]). The CLYC energy resolution of $\sim 27.1 \mathrm{keV}$ FWHM at $663 \mathrm{keV}$ [63] is better than $\mathrm{CeBr}_{3}$, while the sensitivity to gamma-rays 
is lower because of its low density and small average atomic number. The CLYC is assumed as a dual mode detector for gamma-ray and neutron.

The detector sizes were chosen to be 2.5 inch $\phi \times 2.5$ inch $\left(\sim 200 \mathrm{~cm}^{3}\right)$ for HPGe and 3 inch $\phi \times 3$ inch $\left(\sim 350 \mathrm{~cm}^{3}\right)$ for the scintillators. The HPGe size was limited by the refrigerator mass.

\subsubsection{Neutron Detector}

Since neutrons have no electric charge, they are detected by interactions with detector nuclei producing charged particles. Slow (thermal and epithermal) neutrons are detected as a positive $Q$-value, where the $Q$-value is the mass difference in energy scale between the total mass of interacting particles before and after the interaction. Thermal neutrons are mainly detected through neutron absorption by nuclei followed by the emission of charged particles. The cross section of neutron absorption is, in general, a decreasing function of neutron energy, except at the resonance energies, where the cross section is appreciably large. The three isotopes of ${ }^{10} \mathrm{~B},{ }^{6} \mathrm{Li}$, and ${ }^{3} \mathrm{He}$ have particularly large cross sections of neutron reaction and large $Q$-values. Therefore, they are important for thermal neutron detection. On the other hand, fast neutrons may scatter nuclei elastically with an energy ranging from zero to the energy of the neutron. The fast neutrons may be detected by measuring the energy of recoil particles.

The neutron spectrometer was defined as being composed of a lithium glass scintillator (LiG) with a boron loaded plastic scintillator (BLP) or a CLYC. A schematic drawing and the detector sensitivities as a function of the neutron energy are shown in Figure 4. The LiG typically shows high sensitivity to thermal neutron $(<0.5 \mathrm{eV})$. The BLP detects separately epithermal $(0.5 \mathrm{Ev}-500 \mathrm{keV})$ and fast $(>500 \mathrm{keV})$ neutrons by delayed coincidence [8] [15]. In the BLP, neutrons undergoing nuclear capture in the $20 \mathrm{~ns}-2.5 \mu \mathrm{s}$ time window after the hydrogen-nuclei elastic reaction with energy over $100 \mathrm{keV}$ were considered as fast neutrons here. The sensitivity of CLYC in epithermal energy range is lower than the BLP because of its lower cross section of neutron reactors. While the CLYC can separately detect gamma-ray and neutron, it cannot discriminate between fast and epithermal neutrons.

\section{Results and Discussion}

\subsection{Gamma-Ray and Neutron Backgrounds from Spacecraft}

The gamma-ray and neutron spectra emitted from the spacecraft body and its fuels are shown in Figure 5(a) and Figure 5(b), respectively. The gamma-ray and neutron background levels in Figure 5(a) and Figure 5(b) are summarized with those by the Mars Odyssey like spacecraft in Table 3 and Table 4, respectively. Comparing the microsatellite with hydrazine and the Mars Odyssey like spacecraft, the use of microsatellite results in a $\sim 1.5$ - 2 times lower gamma-ray backgrounds due to its small mass. On the other hands, the neutron backgrounds is similar in the epithermal and fast energy range, while that of the Mars 


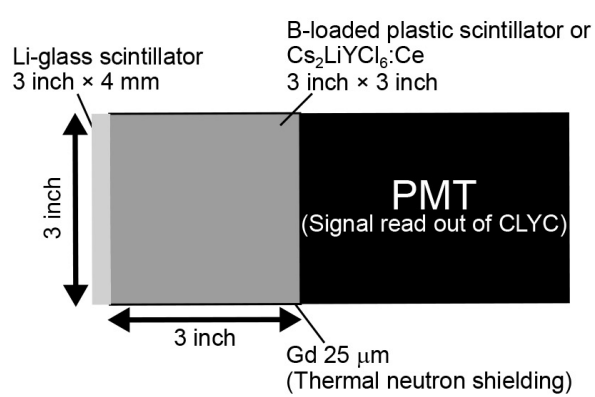

(a)

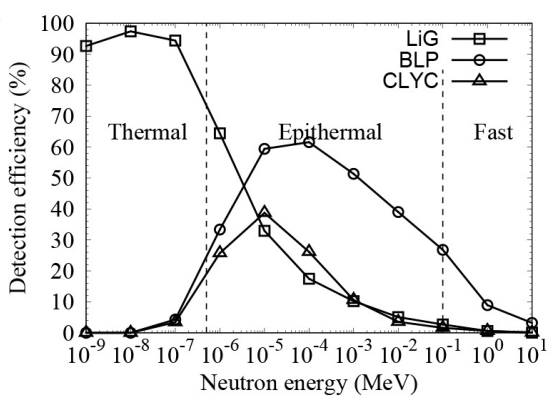

(b)

Figure 4. Schematic drawing of the neutron spectrometer and its sensitivities.
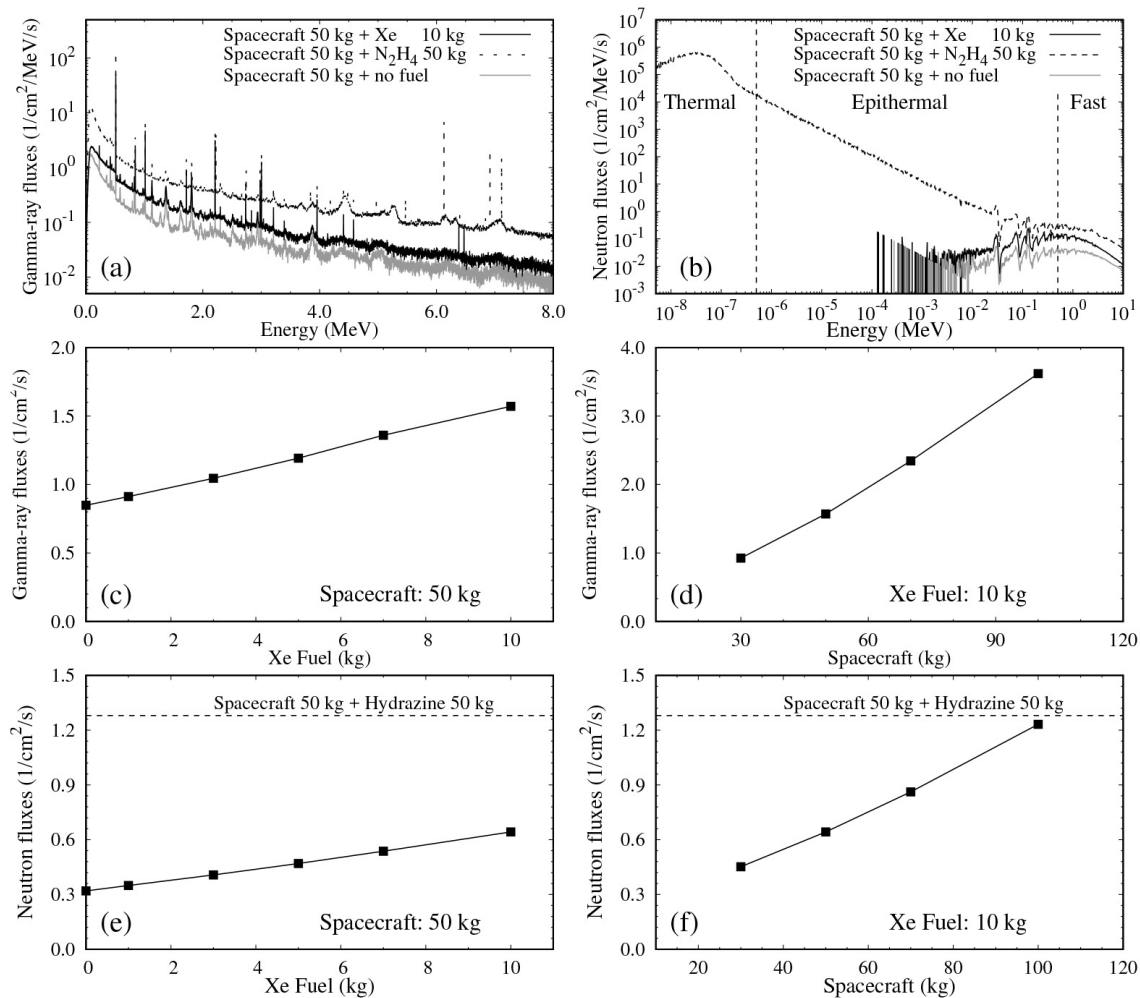

Figure 5. Background gamma-ray fluxes emitted from spacecraft body and its fuels. (a) Energy spectra of gamma-ray emission. (b) Energy spectra of neutron emission. Solid and dot lines represent xenon and hydrazine points, respectively. (c) and (d) Total gamma-ray fluxes at $0-8.0 \mathrm{MeV}$ as a function of xenon fuel and spacecraft mass, respectively. (e) and (f) Total neutron fluxes in $10^{-9}-10 \mathrm{MeV}$ as a function of fuel and spacecraft mass, respectively. The neutron flux by the hydrazine fuel is also shown as a dotted line.

Odyssey like spacecraft in the thermal energy range is larger by a factor of $\sim 1.5$ than the microsatellite one. It is also found that the hydrazine fuel yields a $\sim 3-4$ times larger gamma-ray background than that of the xenon fuel with a similar specific impulse. The hydrazine was assumed to have larger mass to show similar specific impulse with xenon. The larger mass leads the larger gamma-ray background. The differences in the neutron yields are caused by the composition of 
Table 3. Gamma-ray background emission from spacecraft body and fuel (photons $\left./ \mathrm{cm}^{2} / \mathrm{MeV} / \mathrm{min}\right)$.

\begin{tabular}{ccccc}
\hline & $2 \mathrm{MeV}$ & $4 \mathrm{MeV}$ & $6 \mathrm{MeV}$ & $8 \mathrm{MeV}$ \\
\hline No fuel & 3.307 & 1.556 & 0.989 & 0.417 \\
$5 \mathrm{~kg}$ xenon & 4.948 & 2.177 & 1.353 & 0.633 \\
50 kg hydrazine & 23.15 & 9.950 & 5.648 & 3.486 \\
Mars Odyssey & 36.47 & 17.84 & 10.92 & 6.562
\end{tabular}

Table 4. Neutron background emission from spacecraft body and fuel (neutrons $/ \mathrm{cm}^{2} / \mathrm{sec}$ ).

\begin{tabular}{cccc}
\hline & Thermal & Epithermal & Fast \\
\hline No fuel & ---- & 0.015 & 0.305 \\
5 kg xenon & ---- & 0.028 & 0.441 \\
50 kg hydrazine & 0.033 & 0.138 & 1.115 \\
Mars Odyssey & 0.044 & 0.132 & 1.165 \\
\hline
\end{tabular}

fuels as well as its mass. Hydrazine contains a lot of hydrogen which acts as an effective moderator to neutron, shifting fast neutrons induced by GCR interactions to thermal neutrons. Actually, thermal neutron enhancement is visible in the neutron background when using hydrazine as fuel. Therefore, the use of hydrazine fuel produces more gamma-ray and neutron backgrounds than that of xenon fuel.

The emission spectra (Figure 5(a) and Figure 5(b)) have been integrated over the energy to obtain the gamma-ray and neutron emission rates as function of xenon fuel and spacecraft masses (Figures 5(c)-(f)). The neutron emission for a $50 \mathrm{~kg}$ spacecraft body with $50 \mathrm{~kg}$ hydrazine fuel is also shown for comparison in Figure 5(e) and Figure 5(f). As expected, the gamma-ray background increases with the fuel and spacecraft masses. On the other hand, both gamma-ray and neutron backgrounds have never reached the values obtained with hydrazine fuel. These results show that using an ion engine is advantageous for its low background, in particular for gamma-ray spectroscopy where the count rate of some spectral lines is relatively low.

\subsection{Radiations from Celestial Target Bodies}

\subsubsection{Gamma-Ray Emission}

Gamma-ray emission energy spectra from our four types of target compositions are shown in Figure 6. Some typical gamma-ray lines are also assigned in Figure 6 [58] [65]. The differences in elemental compositions are evident in these spectra. Although there are some irregular features such as a gaussian at $\sim 5.2 \mathrm{MeV}$ and rectangles at $\sim 5.5,6.5$, and $7.5 \mathrm{MeV}$, these gamma-rays are emitted by errors in the calculation mechanism of PHITS. The gaussian is derived from oxygen gamma-ray with the energy of $5.269 \mathrm{MeV}$, while the rectangles correspond to 


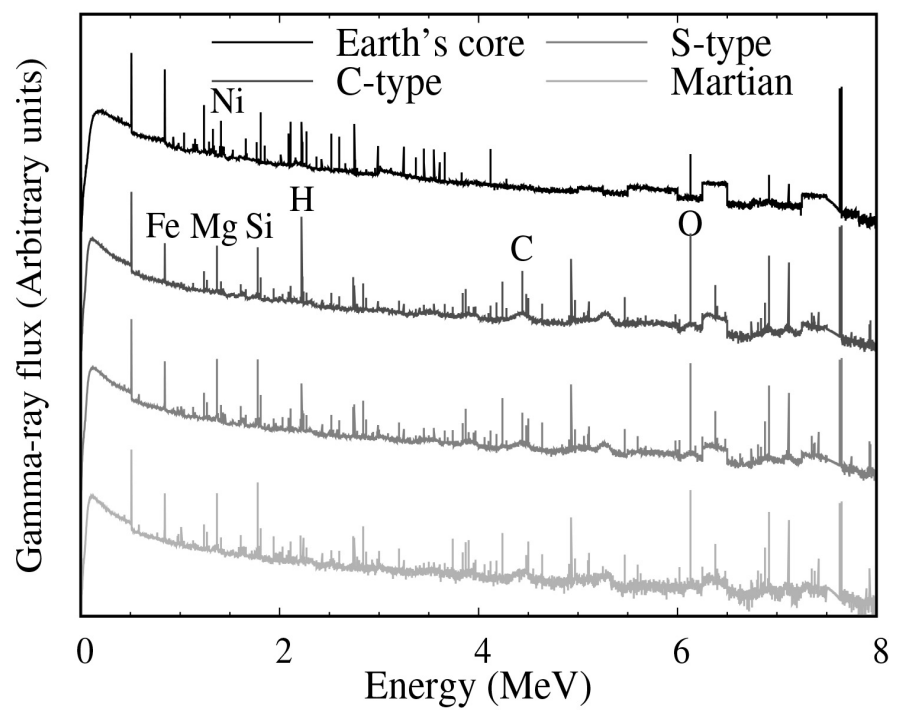

Figure 6. Gamma-ray emission spectra obtained by the four target compositions. Some typical gamma-ray lines are indicated [58] [65].

iron gamma-ray lines at 5.921, 6.382, and 7.509 MeV, respectively [36]. These irregular gamma-rays were integrated with a linear continuum to approach the gamma-ray lines at the corresponding energies. We note that gamma-ray lines from radioactive nuclei decay are not present. The emission rates of some major gamma-ray lines are summarized in Table 5 [58] [65].

The emission rate dependences of some strong gamma-ray lines on the hydrogen concentration are shown in Figure 7 . The values in this figure are normalized at those of $100 \mathrm{ppm}$ hydrogen. Generally, $(\mathrm{n}, \gamma)$ plots increase with the hydrogen concentration, while ( $\mathrm{n}, \mathrm{n} \gamma$ ) plots decrease with the hydrogen concentration. This trend is induced by thermal and fast neutron dependence on the hydrogen concentration, which is described in the next section. The fast neutron decrease leads to a reduction of the inelastic scattering gamma-ray production. The variation of $\mathrm{O}(\mathrm{n}, \mathrm{n} \gamma)$ gamma-rays is small compared with the other plots. We have evaluated the water equivalent hydrogen concentration in the sample, i.e., the number of oxygen nuclei increases in a fixed 1:2 proportion to that of hydrogen nuclei. The fast neutron decrease together with the oxygen nuclei increase induces a balancing effect on the dependence of $\mathrm{O}(\mathrm{n}, \mathrm{n} \gamma)$ gamma-ray. The thermal neutron dependence on the hydrogen concentration leads to an enhancement of the neutron capture gamma-rays for up to $10,000 \mathrm{ppm}$ hydrogen, moderately decreasing at 20,000 ppm hydrogen. The $\mathrm{H}(\mathrm{n}, \gamma)$ plots increase drastically, reaching over 500 at 20,000 ppm hydrogen. This is not only due to the increase of thermal neutron but also of hydrogen concentration.

Major gamma-ray emission rates from radioactive elements with different concentrations for the C-type case are shown in Table 6 [58] [65]. The emission rates with the other elemental compositions were confirmed to be similar to the C-types ones. 
Table 5. Gamma-ray emission rates induced by neutron interactions (photons $/ \mathrm{cm}^{2} / \mathrm{min}$ ) [58] [65].

\begin{tabular}{|c|c|c|c|c|c|c|}
\hline Nuclei & Reaction & Energy $(\mathrm{MeV})$ & Earth's core & C-type & S-type & Martian \\
\hline $\mathrm{H}$ & $(\mathrm{n}, \gamma)$ & 2.223 & 0.343 & 2.473 & 0.306 & ----- \\
\hline $\mathrm{C}$ & $(\mathrm{n}, \mathrm{n} \gamma)$ & 4.440 & ---- & 0.122 & 0.056 & ----- \\
\hline \multirow[t]{3}{*}{$\mathrm{O}$} & $(\mathrm{n}, \mathrm{n} \gamma)$ & 7.117 & 0.098 & 0.209 & 0.193 & 0.215 \\
\hline & $(\mathrm{n}, \mathrm{n} \gamma)$ & 6.916 & 0.018 & 0.306 & 0.291 & 0.308 \\
\hline & $(\mathrm{n}, \mathrm{n} \gamma)$ & 6.129 & 0.064 & 1.012 & 0.974 & 1.097 \\
\hline \multirow[t]{3}{*}{$\mathrm{Mg}$} & $(\mathrm{n}, \mathrm{n} \gamma)$ & 4.238 & ----- & 0.067 & 0.140 & 0.100 \\
\hline & $(\mathrm{n}, \mathrm{n} \gamma)$ & 1.809 & ----- & 0.065 & 0.144 & 0.109 \\
\hline & $(\mathrm{n}, \mathrm{n} \gamma)$ & 1.369 & ---- & 0.481 & 1.168 & 0.373 \\
\hline \multirow[t]{4}{*}{$\mathrm{Al}$} & $(\mathrm{n}, \mathrm{n} \gamma)$ & 3.004 & ---- & ----- & 0.008 & 0.025 \\
\hline & $(\mathrm{n}, \mathrm{n} \gamma)$ & 2.211 & ---- & 0.007 & 0.021 & 0.069 \\
\hline & $(\mathrm{n}, \mathrm{n} \gamma)$ & 1.015 & ----- & 0.014 & 0.030 & 0.090 \\
\hline & $(\mathrm{n}, \mathrm{n} \gamma)$ & 0.844 & ----- & 0.020 & 0.029 & 0.054 \\
\hline \multirow[t]{7}{*}{$\mathrm{Si}$} & $(\mathrm{n}, \mathrm{n} \gamma)$ & 7.416 & ---- & 0.008 & 0.018 & 0.022 \\
\hline & $(\mathrm{n}, \mathrm{n} \gamma)$ & 6.878 & ---- & 0.019 & 0.037 & 0.045 \\
\hline & $(\mathrm{n}, \mathrm{n} \gamma)$ & 5.109 & ----- & 0.010 & 0.023 & 0.028 \\
\hline & $(\mathrm{n}, \mathrm{n} \gamma)$ & 5.099 & ----- & 0.006 & 0.012 & 0.016 \\
\hline & $(\mathrm{n}, \gamma)$ & 4.934 & ---- & 0.253 & 0.305 & 0.246 \\
\hline & $(\mathrm{n}, \mathrm{n} \gamma)$ & 2.839 & ---- & 0.052 & 0.113 & 0.140 \\
\hline & $(\mathrm{n}, \mathrm{n} \gamma)$ & 1.779 & ----- & 0.452 & 1.168 & 1.627 \\
\hline \multirow[t]{5}{*}{$\mathrm{Ca}$} & $(\mathrm{n}, \mathrm{n} \gamma)$ & 3.904 & ---- & 0.007 & 0.009 & 0.061 \\
\hline & $(\mathrm{n}, \mathrm{n} \gamma)$ & 3.737 & ----- & 0.008 & 0.013 & 0.068 \\
\hline & $(\mathrm{n}, \gamma)$ & 1.943 & ---- & 0.025 & 0.022 & 0.067 \\
\hline & $(\mathrm{n}, \mathrm{n} \gamma)$ & 1.611 & ----- & 0.019 & 0.059 & 0.059 \\
\hline & $(\mathrm{n}, \mathrm{n} \gamma)$ & 0.771 & ----- & ----- & 0.005 & 0.031 \\
\hline \multirow[t]{5}{*}{$\mathrm{Fe}$} & $(\mathrm{n}, \gamma)$ & 7.646 & 2.622 & 1.576 & 1.299 & 0.599 \\
\hline & $(\mathrm{n}, \gamma)$ & 7.631 & 2.403 & 1.445 & 1.186 & 0.549 \\
\hline & $(\mathrm{n}, \mathrm{n} \gamma)$ & 1.811 & 0.127 & 0.066 & 0.102 & 0.089 \\
\hline & $(\mathrm{n}, \mathrm{n} \gamma)$ & 1.238 & 0.911 & 0.090 & 0.100 & 0.131 \\
\hline & $(\mathrm{n}, \mathrm{n} \gamma)$ & 0.847 & 6.574 & 0.503 & 1.009 & 0.849 \\
\hline
\end{tabular}

Table 6. Gamma-rays emission rates by radioactive nuclei (photons $/ \mathrm{cm}^{2} / \mathrm{min}$ ) [58] [65]. The concentration units are ppm for potassium, and ppb for thorium and uranium.

\begin{tabular}{cccc}
\hline Concentration & $\mathrm{K} @ 1.46 \mathrm{MeV}$ & $\mathrm{Th} @ 2.61 \mathrm{MeV}$ & $\mathrm{U} @ 609 \mathrm{keV}$ \\
\hline 200 & 0.185 & 0.111 & 0.209 \\
400 & 0.368 & 0.222 & 0.419 \\
600 & 0.552 & 0.332 & 0.629 \\
800 & 0.735 & 0.442 & 0.838 \\
1000 & 0.919 & 0.552 & 1.048 \\
\hline
\end{tabular}




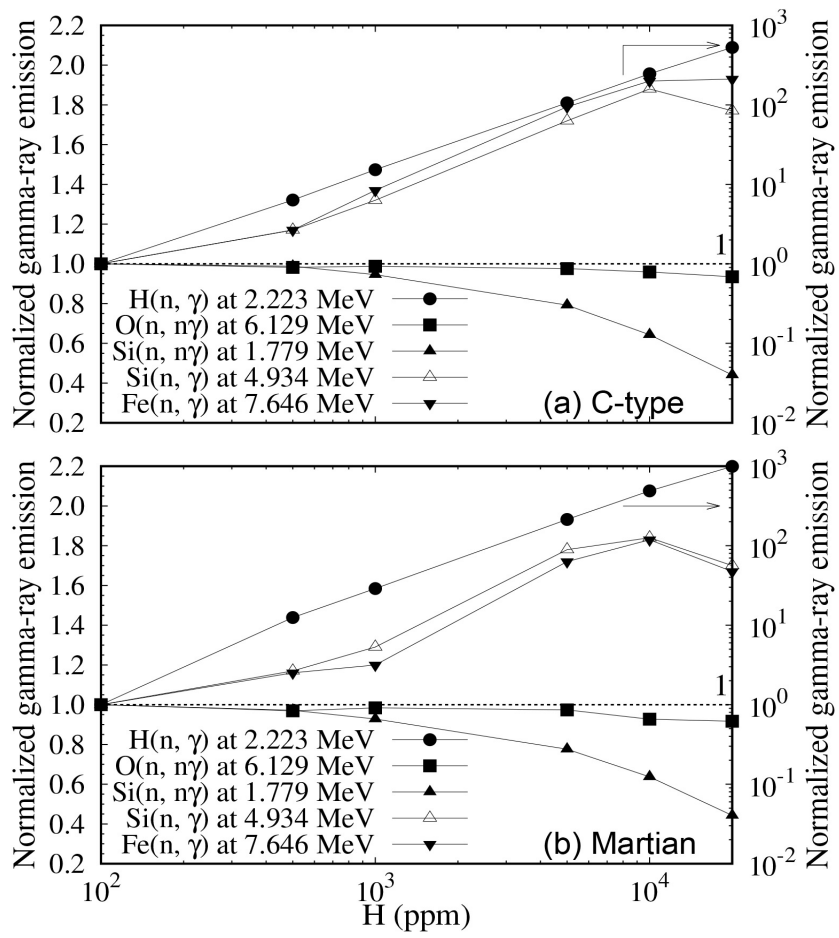

Figure 7. Gamma-ray emission counts as a function of the hydrogen concentration. The values in this figure are normalized at the emission rates of $100 \mathrm{ppm}$ hydrogen. $\mathrm{H}(\mathrm{n}, \gamma)$ plots are presented in log scale while the others are in linear scale.

\subsubsection{Neutron Emission}

As shown in Figure 8, neutron fluxes in the epithermal energy show differences depending on the hydrogen concentration in each composition, while the neutron energy spectra for targets with high hydrogen compositions show peaks in the thermal energy range. The neutron emission rates in the three energy ranges are shown in Table 7. According to Naito et al. [66], the emission rates of epithermal and fast neutrons decrease with hydrogen concentration, while that of thermal neutron becomes maximum at $\sim 2000 \mathrm{ppm}$. Figure 9 shows the neutron emission rate dependence on hydrogen concentration. The values are normalized to the $0 \mathrm{ppm}$ hydrogen case. The neutron emission rate dependence on hydrogen concentration is explained by the moderation and decrease of $\langle A\rangle$. The increase of hydrogen concentration induces the decrease of $\langle A\rangle$, which decreases the neutron yields by spallation. Effective moderation by hydrogen atoms increases the thermal neutron, but for very high hydrogen concentrations it begins to decrease due to low neutron yields. The large dynamic range of epithermal neutron flux is caused by both effective moderation and yield decrease.

\subsection{Detection by Gamma-Ray and Neutron Spectrometer}

\subsubsection{Gamma-Ray Detection}

Figure 10 shows the relative values of $F$ for the four detectors obtained by the $\sqrt{\varepsilon(E) / R(E)}$ relation as a function of gamma-ray energy. The values of 


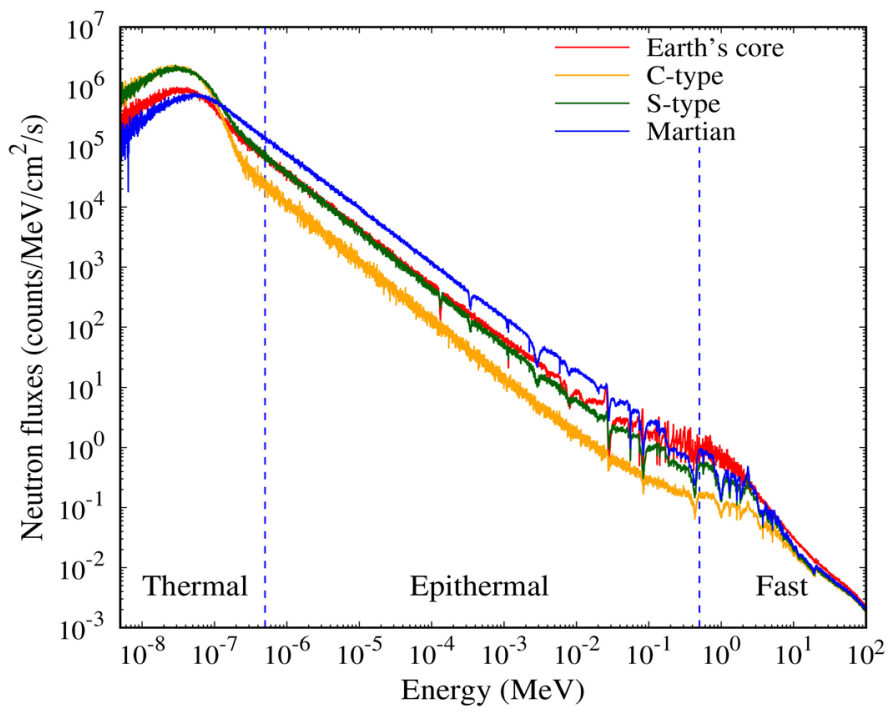

Figure 8. Neutron energy spectra emitted by the four different elemental compositions.

Table 7. Emission rates of thermal, epithermal, and fast neutrons (neutrons $/ \mathrm{cm}^{2} / \mathrm{sec}$ ).

\begin{tabular}{ccccc}
\hline & Earth's core & C-type & S-type & Martian \\
\hline Thermal $(<0.5 \mathrm{eV})$ & 0.147 & 0.185 & 0.238 & 0.217 \\
Epithermal & 1.268 & 0.246 & 0.285 & 1.955 \\
$(0.5 \mathrm{eV}-500 \mathrm{keV})$ & & 0.486 & 1.419 & 1.730 \\
Fast $(>500 \mathrm{keV})$ & 4.580 & & & \\
\hline
\end{tabular}

$\varepsilon(E)$ and $R(E)$ were calculated by simulations and square root law of energy, respectively. The values in Figure 10 are normalized to $F$ obtained with HPGe and $1 \mathrm{MeV}$ gamma-rays. The HPGe detector shows a $\sim 2.5$ times higher value of $F$ than $\mathrm{LaBr}^{3}$ due to its excellent energy resolution. Differences in $F$ between $\mathrm{CeBr}^{3}$ and $\mathrm{LaBr}^{3}$ were $\sim 10 \%$, and those between $\mathrm{CeBr}^{3}$ and CLYC were $\sim 15 \%-40 \%$.

The C-type asteroid gamma-ray spectra of the different detectors are shown in Figure 11. The solid angle parameter $H / R$ was assumed to be 2.0 in this figure. Also in the following figures, $H / R$ was fixed to 2.0 unless noted otherwise. Differences in $F$ appear in the energy spectra. The HPGe spectrum shows sharp gamma-ray lines and low continuum level, which are results of its high energy resolution and low detection efficiency, respectively. The $\mathrm{CeBr}^{3}$ and $\mathrm{LaBr}^{3}(\mathrm{Ce})$ spectra strongly overlap due to the similar detection efficiencies and energy resolutions. The CLYC spectrum shows a similar detection efficiency and energy resolution to HPGe and $\mathrm{CeBr}^{3}$ and $\mathrm{LaBr}^{3}$, respectively. The gamma-ray background spectrum from the spacecraft body detected by the HPGe detector is also shown as a black line in Figure 11(a). It is $\sim 1 / 10$ of the target gamma-ray counts for this solid angle. If the background spectrum is measured during the cruising phase, it will be effectively subtracted from the target measurements. 

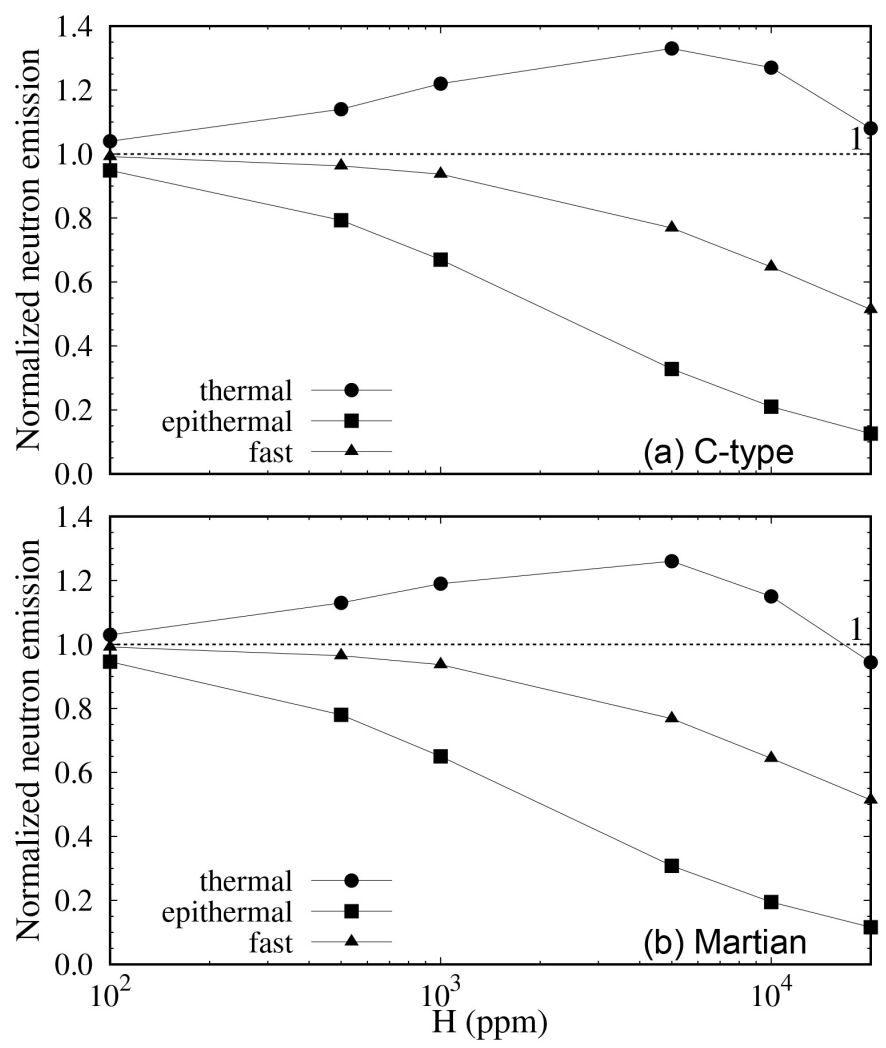

Figure 9. Neutron emission counts as a function of the hydrogen concentration. Values in this figure are normalized to the $0 \mathrm{ppm}$ hydrogen case.

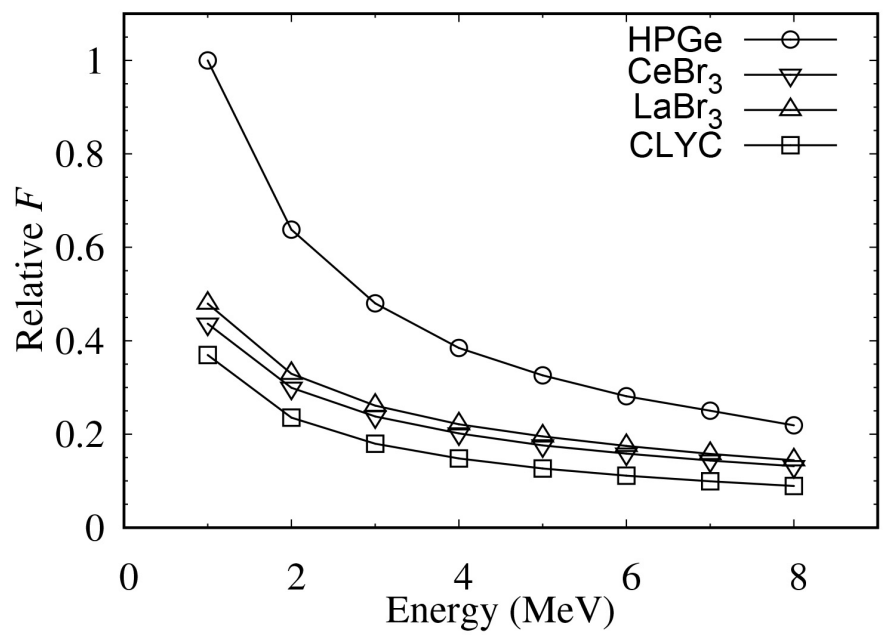

Figure 10. Relative figure of merit $F$ as a function of gamma-ray energy. The values are normalized to $1.0 \mathrm{MeV}$ for HPGe.

Nevertheless, this low background level will not affect significantly the statistical error of target measurement.

The $\operatorname{LaBr}^{3}(\mathrm{Ce})$ internal background gamma-ray spectrum is also shown in Figure 11. Its count rate level is significantly larger than that from the target 


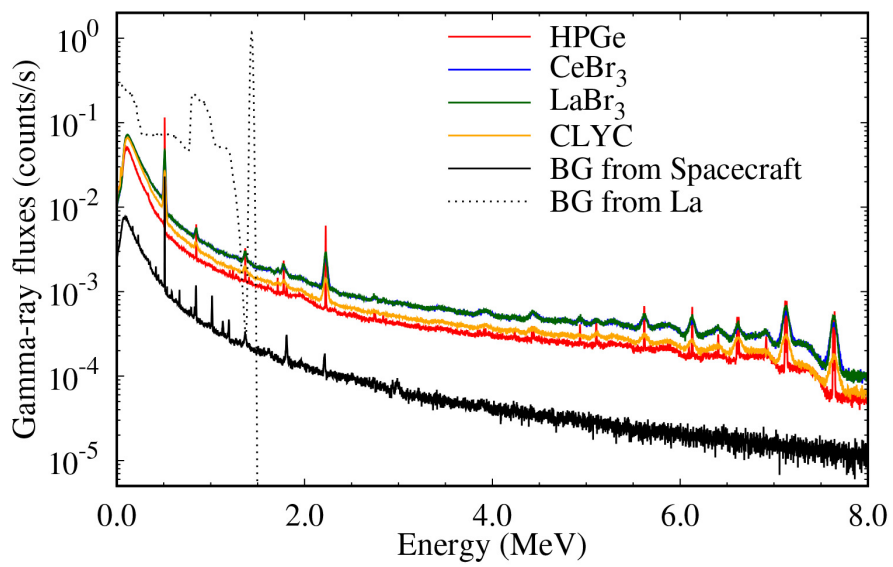

Figure 11. The gamma-ray spectra of C-type composition detected by the four types of detectors for $H / R=2.0$. The detector types are HPGe (red), $\mathrm{CeBr}^{3}$ (blue), $\mathrm{LaBr}^{3}$ (Ce) (green) and CLYC (yellow). The background gamma-ray spectrum from the spacecraft detected by HPGe and $\mathrm{LaBr}^{3}$ internal background are also shown as a black line and a dotted curve, respectively. The differences in detection efficiency and energy resolution appear in the spectra.

measurement one. With this background level it is difficult to detect gamma-ray lines below $1.5 \mathrm{MeV}$. In particular, the lanthanum gamma-ray line at $1.44 \mathrm{MeV}$ makes the estimation of potassium gamma-ray line at $1.46 \mathrm{MeV}$ impossible. Hence, the use of this detector is not an applicable choice.

\subsubsection{Neutron Detection}

Figure 12(a) and Figure 12(b) show the count rates of BLP and CLYC with a LiG, respectively. The neutron count rates generally decrease with hydrogen concentration. This effect is related to the variation in both the neutron emission rate and detector sensitivity. The emission rate of epithermal neutrons decreases with the hydrogen concentration, while thermal neutron emission has a peak at $2000 \mathrm{ppm}$. On the other hand, the LiG is partially sensitive to epithermal neutron as shown in Figure 4 (b) $(\sim 70 \%$ at $1 \mathrm{eV})$. The LiG count rate decreasing with the hydrogen concentration is explained by the energy response of detector sensitivity. Despite the similar LiG configuration between the BLP and CLYC setup, the LiG count rates in Figure 12 show differences. It is considered that some neutron events which returned to the LiG by scattering in the BLP result in higher LiG count rates in Figure 12(a). The count rate of the BLP is larger than that of the CLYC due to higher detection sensitivity (Figure 4(b)).

Comparing the count rates of C-type and Martian composition depending on hydrogen concentration, the count rates of BLP and CLYC seem to depend on the hydrogen concentration and do not distinguishing between the two compositions. This conclusion on the BLP and CLYC count rates can be extended to the S-type composition, which contains $\sim 3300 \mathrm{ppm}$ hydrogen. However, the plot corresponding to Earth's core composition does not match these trends. The 


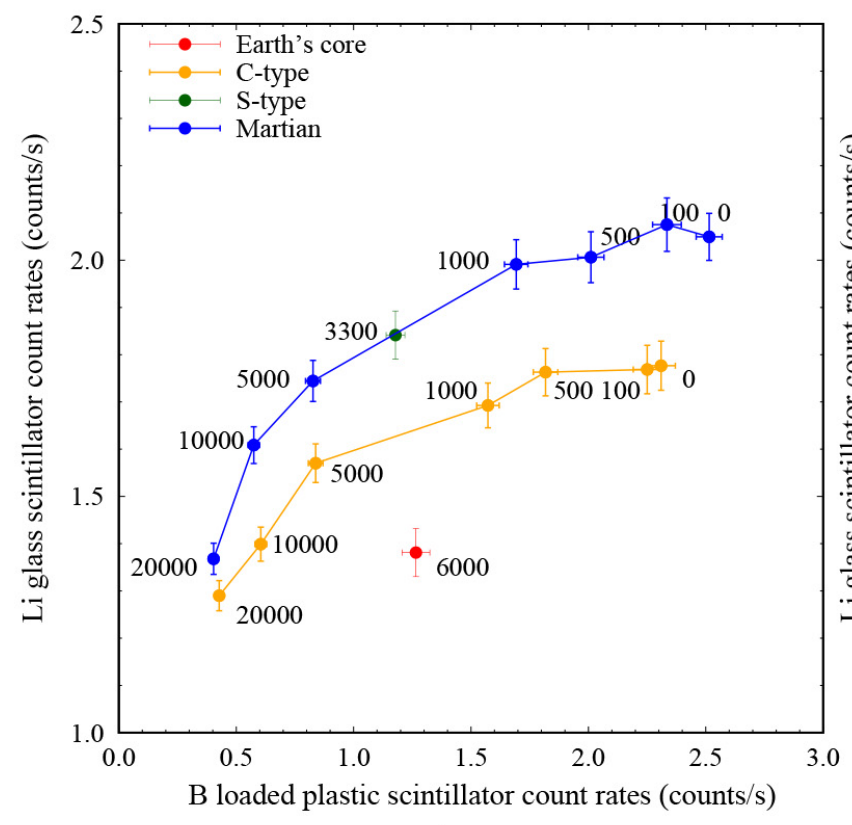

(a)

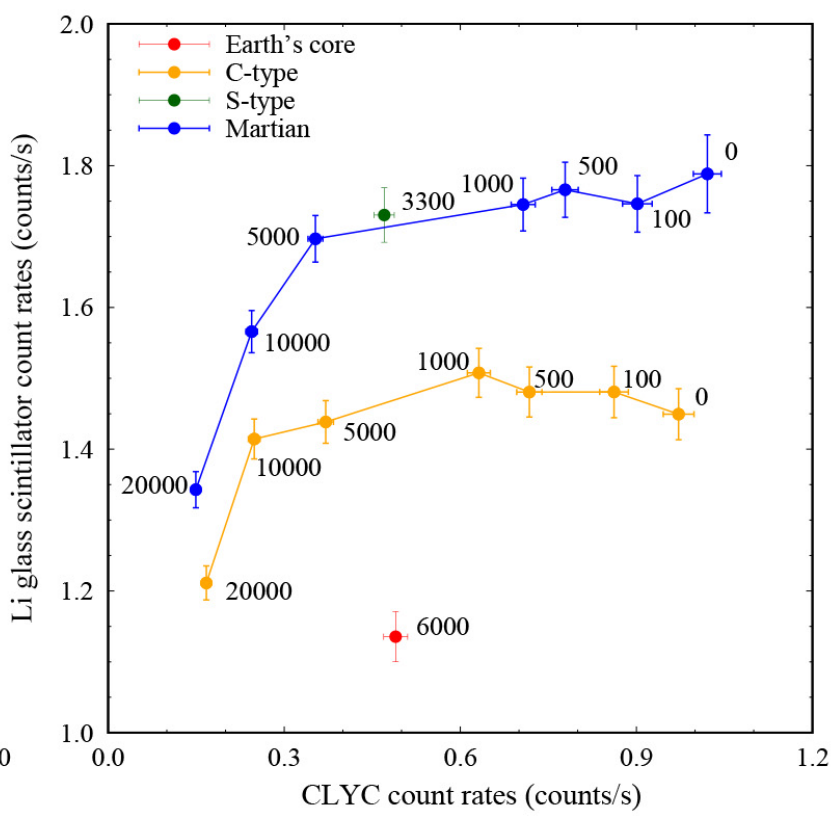

(b)

Figure 12. Count rates of the BLP and CLYC with LiG ((a) and (b), respectively). The values in these figures represent hydrogen concentrations in samples.

core composition with high iron concentration may be the cause for these abnormal values. A material with large average atomic mass produces a large number of neutrons, but its moderation is not effective. Actually, high fast neutron count rates are shown in shown in Figure 12(b) as a consequence of the large average atomic mass. Moreover, the relatively large neutron capture cross section of iron results in a low thermal neutron flux. Thermal neutron absorption by iron is also visible in the LiG count rate difference between C-type and Martian compositions. Therefore, it is reasonable to conclude that the core composition does not provide sufficient moderation enough for the production of thermal and epithermal neutrons.

The CLYC count rates show larger differences depending on hydrogen concentration than the BLP count rates, especially in the range of $0-500 \mathrm{ppm}$. In the present simulation, the neutron count rate identification between 0 and 100 ppm of hydrogen requires 3600 and 300 seconds for BLP and CLYC, respectively. In this respect, the CLYC has a higher potential for the hydrogen detection than the BLP although its count rates are lower. Here the value will change with the solid angle shown in Figure 2, i.e., the minimum acquisition time becomes four times longer in the case of $H / R=2.5$. However, as mentioned in Section 2.2.2, the CLYC is not usable for the discrimination between fast and epithermal neutrons.

\subsection{Detector Selection}

According to the above sections, the best selection of detectors for microsatellite 
missions is discussed here.

From the point of view of gamma-ray detection, the HPGe is the best detector (Figure 10). However, it requires a refrigerator and an annealing heater. The HPGe is an applicable choice when masses, power consumptions and thermal flow are acceptable, but it might be practically difficult for microsatellite missions.

The dual mode CLYC detector for gamma-rays and neutrons has strong advantages in mass and electric power consumption. Although its gamma-ray sensitivity is a little lower than the other detectors, these advantages are quite important for microsatellite missions. The CLYC has such a high potential of hydrogen detection that it is a powerful tool for the exploration of space resources. On the other hand, the CLYC cannot detect fast neutron, which is required for the (n, $\mathrm{n} \gamma$ ) gamma-ray correction. If the CLYC detector is selected, the elemental concentrations should be determined by the $(\mathrm{n}, \gamma)$ lines. Otherwise, the elemental ratios can be determined by the ( $\mathrm{n}, \mathrm{n} \gamma$ ) gamma-rays, similar to the Apollo mission X-ray experiments [23] [24].

If a detailed observation of fast neutron is required, a combined BLP and LiG should be employed. This is the case when the determination of elemental abundances is based on the (n, $\mathrm{n} \gamma)$ lines, and the observation target is the M-type asteroid showing typical neutron fluxes. For the hydrogen determination of M-type asteroids, more numerical estimations with different elemental compositions need to be conducted. When the BLP and LiG are used as a neutron spectrometer, the $\mathrm{CeBr}^{3}$ detector with higher figure of merit is recommended as a gamma-ray spectrometer rather than the CLYC detector.

\section{Conclusions}

The exploration of NEAs is attractive from the points of view of scientific observation and sustainability to maintain human activity because they provide massive storages of valuable resources. Microsatellite programs have recently become a worldwide space activity. Low cost, short time delivery, and innovation in technology are tangible advantages of the microsatellite deep space missions. Microsatellites with ion-propulsion engine open a new window to investigate NEAs.

We assessed the performance of gamma-ray and neutron spectroscopy on microsatellite missions based on numerical simulations. A microsatellite yields over 1.5 - 2.0 times less gamma-ray and neutron backgrounds when compared to a previous large scale spacecraft like Mars Odyssey. The background fluxes emitted from a microsatellite with xenon fuel is $\sim 3-10$ times lower than the fluxes of target planetary bodies in the case of $H / R=2.0$. This low background counting rate is a great advantage for nuclear spectroscopy to be performed onboard the microsatellite. The four target elemental compositions considered in this work were Earth's core, C-type and S-type chondrites, and Martian meteorite, each showing unique features in gamma-ray and neutron emis- 
sion rates and their energy spectra. Observation by the nuclear spectrometer is able to determine the elemental compositions of these targets. The dependence of neutron fluxes on the hydrogen content was confirmed to be evident. Gamma-rays induced by the neutron interactions were also found to clearly depend on the hydrogen concentration. These dependences hydrogens are explained by the effective neutron attenuation of hydrogen atom in the planetary surface material.

Four types of gamma-ray detectors, i.e., HPGe, $\mathrm{CeBr}^{3}, \mathrm{LaBr}^{3}$, and CLYC, and two types of neutron detector configurations, i.e., LiG with BLP or CLYC were compared for the evaluation of potential for the determination of elemental abundances. Each detector showed different advantages and disadvantages. The HPGe has the highest figure of merit $F$, while the CLYC shows advantages in mass and power consumption because of its sensitivity to both gamma-ray and neutron. The BLP with sensitivity to fast neutrons shows high counting rates. On the other hand, the CLYC has higher potential of hydrogen discrimination in low concentration despite its lower count rates. The count rate of $\mathrm{LiG}$ detector highly depends on the elemental composition, because the neutron capture cross sections differ across individual elements. Combining these detectors will allow for obtaining not only the hydrogen concentration but also the rough elemental composition of the target asteroid.

From the points of view of payload for the microsatellite missions, the CLYC combining with LiG appears to be the most promising spectrometer. However, by considering the actual constraints on mass, power consumption and thermal condition as well as mission objectives, the optimal setup of detector system should be selected to meet the specific mission requirement.

\section{Acknowledgements}

This paper is a part of the outcome research performed under a Waseda University Grant for Special Research Project (Project number: 2017B-208).

\section{Conflicts of Interest}

The authors declare no conflicts of interest regarding the publication of this paper.

\section{References}

[1] Funase, R., Koizumi, H., Nakasuka, S., Kawakatsu, Y., Fukushima, Y., Tomiki, A., Kobayashi, Y., Mita, M., Kobayashi, D., Nonomura, T., Science, A. and Agency, E. (2014) 50 kg-Class Deep Space Exploration Technology Demonstration Micro-Spacecraft. 28th Annual AIAA/USU Conf. Small Satellites, Logan, UT, 2-7 August 2014, 3-6.

[2] Tsuruda, Y., Aoyanagi, Y., Tanaka, T., Matsumoto, T., Nakasuka, S., Shirasaka, S., Matsui, M. and Mase, I. (2015) Demonstration of Innovative System Design for Twin Micro-Satellite: Hodoyoshi-3 and -4. Trans. JSASS, 14, 131-140.

[3] Hasebe, N., Kusano, H., Nagaoka, H., Shimizu, S., Miyajima, M., Shibamura, E., 
Kuno, H., Naito, M., Yoshida, K., Adachi, T. and Matias Lopes, J.A. (2015) Nuclear Spectroscopic Approach to Study M-Type Near-Earth-Asteroids on the Miniature Deep Space Satellite. Proc. ISTS 30 th, Kobe, Hyogo, 4-10 July 2015, 2015-f-24.

[4] Hasebe, N., Kim, K.J., Shibamura, E. and Sakurai, K. (2017) Nuclear Planetary Science: Planetary Science Based on Gamma-Ray, Neutron, and X-Ray Spectroscopy. World Sciectific, Singapore. https://doi.org/10.1142/10439

[5] Harrington, T.M., Marshall, J.H., Arnold, J.R., Peterson, L.E., Trombka, J.I. and Metzger, A.E. (1974) The Apollo Gamma-Ray Spectrometer. Nuclear Instruments \& Methods in Physics Research Section A, 118, 401-411. https://doi.org/10.1016/0029-554X(74)90644-2

[6] Goldsten, J.O., McNutt, R.L., Gold, R.E., Gary, S.A., Fiore, E., Schneider, S.E., Hayes, J.R., Trombka, J.I., Floyd, S.R., Boynton, W.V., Bailey, S., Bruckner, J., Squyres, S.W., Evans, L.G., Clark, P.E. and Starr, R. (1997) The X-Ray/Gamma-Ray Spectrometer on the Near Earth Asteroid Rendezvous Mission. Space Science Reviews, 82, 169-216. https://doi.org/10.1023/A:1005052720796

[7] Feldman, W.C., Barraclough, B.L., Fuller, K.R., Lawrence, D.J., Maurice, S., Miller, M.C., Prettyman, T.H. and Binder, A.B. (1999) The Lunar Prospector Gamma-Ray and Neutron Spectrometers. Nuclear Instruments \& Methods in Physics Research Section A, 422, 562-566. https://doi.org/10.1016/S0168-9002(98)00934-6

[8] Boynton, W.V., Feldman, W.C., Mitrofanov, I.G., Evans, L.G., Reedy, R.C., Squyres, S.W., Starr, R., Trombka, J.I., D’uston, C., Arnold, J.R., Englert, P.A.J., Metzger, A.E., Wänke, H., Brückner, J., Drake, D.M., Shinohara, C., Fellows, C., Hamara, D.K., Harshman, K., Kerry, K., Turner, C., Ward, M., Barthe, H., Fuller, K.R., Storms, S.A., Thornton, G.W., Longmire, J.L., Litvak, M.L. and Ton'chev, A.K. (2004) The Mars Odyssey Gamma-Ray Spectrometer Instrument Suite. Space Science Reviews, 110, 37-83. https://doi.org/10.1023/B:SPAC.0000021007.76126.15

[9] Hasebe, N., Shibamura, E., Miyachi, T., Takashima, T., Kobayashi, M., Okudaira, O., Yamashita, N., Kobayashi, S., Ishizaki, T., Sakurai, K., Miyajima, M., Fujii, M., Narasaki, K., Takai, S., Tsurumi, K., Kaneko, H., Nakazawa, M., Mori, K., Gasnault, O., Maurice, S., D'Uston, C., Reedy, R.C. and Grande, M. (2008) Gamma-Ray Spectrometer (GRS) for Lunar Polar Orbiter SELENE. Earth Planets Space, 60, 299-312. https://doi.org/10.1186/BF03352795

[10] Chang, J., Ma, T., Zhang, N., Cai, M.S., Gong, Y.Z., Tang, H.S., Zhang, R.J., Wang, N.S., Yu, M., Mao, J.P., Zhou, Y.L., Liu, J.Z., Xu, A.A. and Liu, L.G. (2009) Gamma-Ray Detector on Board Lunar Mission Chang'e-1. Journal of the Physical Society of Japan, 78, 26-28. https://doi.org/10.1143/JPSJS.78SA.26

[11] Zhu, M.H., Ma, T. and Chang, J. (2010) Chang'E-1 Gamma Ray Spectrometer and Preliminary Radioactive Results on the Lunar Surface. Planetary and Space Science, 58, 1547-1554. https://doi.org/10.1016/j.pss.2010.07.022

[12] Goldsten, J.O., Rhodes, E.A., Boynton, W.V., Feldman, W.C., Lawrence, D.J., Trombka, J.I., Smith, D.M., Evans, L.G., White, J., Madden, N.W., Berg, P.C., Murphy, G.A., Gurnee, R.S., Strohbehn, K., Williams, B.D., Schaefer, E.D., Monaco, C.A., Cork, C.P., Del Eckels, J., Miller, W.O., Burks, M.T., Hagler, L.B., DeTeresa, S.J. and Witte, M.C. (2007) The MESSENGER Gamma-Ray and Neutron Spectrometer. Space Science Reviews, 131, 339-391. https://doi.org/10.1007/s11214-007-9262-7

[13] Mitrofanov, I.G., Bartels, A., Bobrovnitsky, Y.I., Boynton, W., Chin, G., Enos, H., Evans, L., Floyd, S., Garvin, J., Golovin, D.V., Grebennikov, A.S., Harshman, K., Kazakov, L.L., Keller, J., Konovalov, A.A., Kozyrev, A.S., Krylov, A.R., Litvak, M.L., 
Malakhov, A.V., McClanahan, T., Milikh, G.M., Mokrousov, M.I., Ponomareva, S., Sagdeev, R.Z., Sanin, A.B., Shevchenko, V.V., Shvetsov, V.N., Starr, R., Timoshenko, G.N., Tomilina, T.M., Tretyakov, V.I., Trombka, J., Troshin, V.S., Uvarov, V.N., Varennikov, A.B. and Vostrukhin, A.A. (2010) Lunar Exploration Neutron Detector for the NASA Lunar Reconnaissance Orbiter. Space Science Reviews, 150, 183-207. https://doi.org/10.1007/s11214-009-9608-4

[14] Mitrofanov, I.G., Litvak, M.L., Varenikov, A.B., Barmakov, Y.N., Behar, A., Bobrovnitsky, Y.I., Bogolubov, E.P., Boynton, W.V., Harshman, K., Kan, E., Kozyrev, A.S., Kuzmin, R.O., Malakhov, A.V., Mokrousov, M.I., Ponomareva, S.N., Ryzhkov, V.I., Sanin, A.B., Smirnov, G.A., Shvetsov, V.N., Timoshenko, G.N., Tomilina, T.M., Tret'yakov, V.I. and Vostrukhin, A.A. (2012) Dynamic Albedo of Neutrons (DAN) Experiment Onboard NASA's Mars Science Laboratory. Space Science Reviews, 170, 559-582. https://doi.org/10.1007/s11214-012-9924-y

[15] Prettyman, T.H., Feldman, W.C., McSween, H.Y., Dingler, R.D., Enemark, D.C., Patrick, D.E., Storms, S.A., Hendricks, J.S., Morgenthaler, J.P., Pitman, K.M. and Reedy, R.C. (2011) Dawn's Gamma Ray and Neutron Detector. Space Science Reviews, 163, 371-459. https://doi.org/10.1007/s11214-011-9862-0

[16] Nozette, S., Rustan, P., Pleasance, L.P., Kordas, J.F., Lewis, I.T., Park, H.S., Priest, R.E., Horan, D.M., Regeon, P., Lichtenberg, C.L., Shoemaker, E.M., Eliason, E.M., McEwen, A.S., Robinson, M.S., Spudis, P.D., Acton, C.H., Buratti, B.J., Duxbury, T.C., Baker, D.N., Jakosky, B.M., Blamont, J.E., Corson, M.P., Resnick, J.H., Rollins, C.J., Davies, M.E., Lucey, P.G., Malaret, E., Massie, M.A., Pieters, C.M., Reisse, R.A., Simpson, R.A., Smith, D.E., Sorenson, T.C., Breugge, R.W. and Zuber, M.T. (1994) The Clementine Mission to the Moon: Scientific Overview. Science, 266, 1835-1839. https://doi.org/10.1126/science.266.5192.1835

[17] Prettyman, T.H., Mittlefehldt, D.W., Yamashita, N., Lawrence, D.J., Beck, A.W., Feldman, W.C., McCoy, T.J., McSween, H.Y., Toplis, M.J., Titus, T.N., Tricarico, P., Reedy, R.C., Hendricks, J.S., Forni, O., Le Corre, L., Li, J.-Y., Mizzon, H., Reddy, V., Raymond, C.A. and Russell, C.T. (2012) Elemental Mapping by Dawn Reveals Exogenic H in Vesta's Regolith. Science, 338, 242-246. https://doi.org/10.1126/science.1225354

[18] Gasnault, O., Feldman, W.C., Maurice, S., Genetay, I., D’Uston, C., Prettyman, T.H. and Moore, K.R. (2001) Composition from Fast Neutrons: Application to the Moon. Geophysical Research Letters, 28, 3797-3800. https://doi.org/10.1029/2001GL013072

[19] Gasnault, O., D’Uston, C., Feldman, W.C. and Maurice, S. (2000) Lunar Fast Neutron Leakage UX Calculation and Its Elemental Abundance Dependence. Journal of Geophysical Research, 105, 4263-4271. https://doi.org/10.1029/1999JE001124

[20] Yamashita, N., Hasebe, N., Miyachi, T., Kobayashi, M., Okudaira, O., Kobayashi, S., Ishizaki, T., Sakurai, K., Miyajima, M., Reedy, R.C., D’Uston, C., Maurice, S. and Gasnault, O. (2008) Complexities of Gamma-Ray Line Intensities from the Lunar Surface. Earth Planets Space, 60, 313-319. https://doi.org/10.1186/BF03352796

[21] Lawrence, D.J., Miller, R.S., Ozimek, M.T., Peplowski, P.N. and Scott, C.J. (2015) High-Resolution Mapping of Lunar Polar Hydrogen with a Low-Resource Orbital Mission. Acta Astronautica, 115, 452-462. https://doi.org/10.1016/j.actaastro.2015.06.010

[22] Feldman, W.C. (1998) Fluxes of Fast and Epithermal Neutrons from Lunar Prospector: Evidence for Water Ice at the Lunar Poles. Science, 281, 1496-1500. https://doi.org/10.1126/science.281.5382.1496 
[23] Adler, I., Trombka, J., Gerard, J., Lowman, P., Schmadebeck, R., Blodget, H., Eller, E., Yin, L., Lamothe, R., Gorenstein, P. and Bjorkholm, P. (1972) Apollo 15 Geochemical X-Ray Fluorescence Experiment: Preliminary Report. Science, 175, 436-440. https://doi.org/10.1126/science.175.4020.436

[24] Adler, I., Trombka, J.I., Gerard, J., Lowman, P., Schmadebeck, R., Blodget, H., Eller, E., Yin, L., Lamothe, R., Osswald, G., Gorenstein, P., Bjorkholm, P., Gursky, H. and Harris, B. (1972) Apollo 16 Geochemical X-Ray Fluorescence Experiment: Preliminary Report. Science, 177, 256-259. https://doi.org/10.1126/science.177.4045.256

[25] Lucey, P.G., Blewett, D.T. and Jolli, B.L. (2000) Lunar Iron and Titanium Abundance Algorithms Based on Final Processing of Clementine Ultraviolet-Visible Images. Journal of Geophysical Research, 105, 20297-20305. https://doi.org/10.1029/1999JE001117

[26] Shkuratov, Y.G., Kaydash, V.G., Stankevich, D.G., Starukhina, L.V., Pinet, P.C., Chevrel, S.D. and Daydou, Y.H. (2005) Derivation of Elemental Abundance Maps at Intermediate Resolution from Optical Interpolation of Lunar Prospector Gamma-Ray Spectrometer Data. Planetary and Space Science, 53, 1287-1301. https://doi.org/10.1016/j.pss.2005.07.001

[27] Wöhler, C., Berezhnoy, A. and Evans, R. (2011) Estimation of Elemental Abundances of the Lunar Regolith Using Clementine UVVIS + NIR Data. Planetary and Space Science, 59, 92-110. https://doi.org/10.1016/j.pss.2010.10.017

[28] Wöhler, C., Grumpe, A., Berezhnoy, A., Bhatt, M.U. and Mall, U. (2014) Integrated Topographic, Photometric and Spectral Analysis of the Lunar Surface: Application to Impact Melt Ows and Ponds. Icarus, 235, 86-122. https://doi.org/10.1016/j.icarus.2014.03.010

[29] Sato, T., Niita, K., Matsuda, N., Hashimoto, S., Iwamoto, Y., Noda, S., Ogawa, T., Iwase, H., Nakashima, H., Fukahori, T., Okumura, K., Kai, T., Chiba, S., Furuta, T. and Sihver, L. (2013) Particle and Heavy Ion Transport Code System, PHITS, Version 2.52. Journal of Nuclear Science and Technology, 50, 913-923. https://doi.org/10.1080/00223131.2013.814553

[30] Nara, Y., Otuka, N., Ohnishi, A., Niita, K. and Chiba, S. (1999) Relativistic Nuclear Collisions at $10 \mathrm{~A} \mathrm{GeV}$ Energies from $\mathrm{p}+\mathrm{Be}$ to $\mathrm{Au}+\mathrm{Au}$ with the Hadronic Cascade Model. Physical Review C, 61, 24901. https://doi.org/10.1103/PhysRevC.61.024901

[31] Boudard, A., Cugnon, J., David, J.-C., Leray, S. and Mancusi, D. (2013) New Potentialities of the Liège Intranuclear Cascade Model for Reactions Induced by Nucleons and Light Charged Particles. Physical Review C, 87, 14606.

https://doi.org/10.1103/PhysRevC.87.014606

[32] Shibata, K., Iwamoto, O., Nakagawa, T., Iwamoto, N., Ichihara, A., Kunieda, S., Chiba, S., Furuhata, K., Otuka, N., Ohasawa, T., Murata, T., Matsunobu, H., Zukeran, A., Kamada, S. and Katakura, J. (2011) JENDL-4.0: A New Library for Nuclear Science and Engineering. Journal of Nuclear Science and Technology, 48, 1-30. https://doi.org/10.1080/18811248.2011.9711675

[33] Niita, K., Chiba, S., Maruyama, T., Maruyama, T., Takada, H., Fukahori, T., Nakahara, Y. and Iwamoto, A. (1995) Analysis of the (N, xN') Reactions by Quantum Molecular Dynamics plus Statistical Decay Model. Physical Review C, 52, 2620-2635. https://doi.org/10.1103/PhysRevC.52.2620

[34] Furihata, S. (2000) Statistical Analysis of Light Fragment Production from Medium Energy Proton-Induced Reactions. Nuclear Instruments and Methods, B171, 251-258. https://doi.org/10.1016/S0168-583X(00)00332-3 
[35] Rawlin, V.K. and Majcher, G.A. (1991) Mass Comparison of Electric Propulsion Systems for NSSK of Geosynchronous Spacecraft.

[36] Saunders, R.S., Arvidson, R.E., Badhwar, G.D., Boynton, W.V., Christensen, P.R., Cucinotta, F.A., Feldman, W.C., Gibbs, R.G., Kloss, C., Landano, M.R., Mase, R.A., McSmith, G.W., Meyer, M.A., Mitrofanov, I.G., Pace, G.D., Plaut, J.J., Sidney, W.P., Spencer, D.A., Thompson, T.W. and Zeitlin, C.J. (2001) Mars Odyssey Mission Summary. Space Science Reviews, 110, 1-36. https://doi.org/10.1023/B:SPAC.0000021006.84299.18

[37] Tholen, D.J. (1989) Asteroid Taxonomic Classifications. University of Arizona Press, Arizona, 1139-1150.

[38] Hardersen, P., Gaffey, M. and Abell, P. (2005) Near-IR Spectral Evidence for the Presence of Iron-Poor Orthopyroxenes on the Surfaces of Six M-Type Asteroids. Icarus, 175, 141-158. https://doi.org/10.1016/j.icarus.2004.10.017

[39] Yoshikawa, M. (2011) Outline of the Next Asteroid Sample Return Mission of Japan: Hayabusa-2. The 28th International Symposium on Space Technology and Science, Cologne, 13-15 March 2017, 2011-2019.

[40] Zindler, A. and Hart, S. (1986) Chemical Geodynamics. Annual Review of Earth and Planetary Sciences, 14, 493-571. https://doi.org/10.1146/annurev.ea.14.050186.002425

[41] Anders, E. and Grevesse, N. (1989) Abundances of the Elements: Meteoritic and Solar. Geochimica et Cosmochimica Acta, 53, 197-214. https://doi.org/10.1016/0016-7037(89)90286-X

[42] Jarosewich, E. (1990) Chemical Analyses of Meteorites: A Compilation of Stony and Iron Meteorite Analyses. Meteoritics, 25, 323-337. https://doi.org/10.1111/j.1945-5100.1990.tb00717.x

[43] Zeigler, R.A. (2015) The Martian Meteorite Compendium. https://curator.jsc.nasa.gov/antmet/mmc/

[44] McSween, H.Y., Grove, T.L. and Wyatt, M.B. (2003) Constraints on the Composition and Petrogenesis of the Martian Crust. Journal of Geophysical Research, 108, 5135. https://doi.org/10.1029/2003JE002175

[45] Lal, D. (1988) Theoretically Expected Variations in the Terrestrial Cosmic-Ray Production Rates of Isotopes. In: Proceedings of the International School of Physics "Enrico Fermi", Italian Physical Society, Amsterdam, 216-233.

[46] Masarik, J. and Reedy, R.C. (1994) Effects of Bulk Composition on Nuclide Production Processes in Meteorites. Geochimica et Cosmochimica Acta, 58, 5307-5317. https://doi.org/10.1016/0016-7037(94)90314-X

[47] Adriani, O., Barbarino, G.C., Bazilevskaya, G.A., Bellotti, R., Boezio, M., Bogomolov, E.A., Bongi, M., Bonvicini, V., Borisov, S., Bottai, S., Bruno, A., Cafagna, F., Campana, D., Carbone, R., Carlson, P., Casolino, M., Castellini, G., Danilchenko, I.A., De Pascale, M.P., De Santis, C., De Simone, N., Di Felice, V., Formato, V., Galper, A.M., Karelin, A.V., Koldashov, S.V., Koldobskiy, S., Krutkov, S.Y., Kvashnin, A.N., Leonov, A., Malakhov, V., Marcelli, L., Mayorov, A.G., Menn, W., Mikhailov, V.V., Mocchiutti, E., Monaco, A., Mori, N., Nikonov, N., Osteria, G., Palma, F., Papini, P., Pearce, M., Picozza, P., Pizzolotto, C., Ricci, M., Ricciarini, S.B., Rossetto, L., Sarkar, R., Simon, M., Sparvoli, R., Spillantini, P., Stozhkov, Y.I., Vacchi, A., Vannuccini, E., Vasilyev, G., Voronov, S.A., Yurkin, Y.T., Wu, J., Zampa, G., Zampa, N. and Zverev, V.G. (2013) Measument of the Isotoropic Composition of Hydrogen and Helium Nuclei in Cosmic Rays with the PAMELA Experiment. The 
Astrophysical Journal, 770, 2. https://doi.org/10.1088/0004-637X/770/1/2

[48] Adriani, O., Barbarino, G.C., Bazilevskaya, G.A., Bellotti, R., Boezio, M., Bogomolov, E.A., Bongi, M., Bonvicini, V., Bottai, S., Bruno, A., Cafagna, F., Campana, D., Carlson, P., Casolino, M., Castellini, G., Donato, C.D., Santis, C.D., Simone, N.D., Felice, V.D., Formato, V., Galper, A.M., Karelin, A.V., Koldashov, S.V., Koldobskiy, S., Krutkov, S.Y., Kvashnin, A.N., Leonov, A., Malakhov, V., Marcelli, L., Martucci, M., Mayorov, A.G., Menn, W., Mergè, M., Mikhailov, V.V., Mocchiutti, E., Monaco, A., Mori, N., Munini, R., Osteria, G., Palma, F., Panico, B., Papini, P., Pearce, M., Picozza, P., Ricci, M., Ricciarini, S.B., Sarkar, R., Scotti, V., Simon, M., Sparvoli, R., Spillantini, P., Stozhkov, Y.I., Vacchi, A., Vannuccini, E., Vasilyev, G., Voronov, S.A., Yurkin, Y.T., Zampa, G. and Zampa, N. (2016) Measurement of Cosmic-Ray Hydrogen and Helium Isotopes with the PAMELA Experiment. The Astrophysical Journal, 818, 68. https://doi.org/10.3847/0004-637X/818/1/68

[49] Usoskin, I.G., Bazilevskaya, G.A. and Kovaltsov, G.A. (2011) Solar Modulation Parameter for Cosmic Rays since 1936 Reconstructed from Ground-Based Neutron Monitors and Ionization Chambers. Journal of Geophysical Research, 116, Article ID: 02104. https://doi.org/10.1029/2010JA016105

[50] Shikaze, Y., Haino, S., Abe, K., Fuke, H., Hams, T., Kim, K.C., Makida, Y., Matsuda, S., Mitchell, J.W., Moiseev, A.A., Nishimura, J., Nozaki, M., Orito, S., Ormes, J.F., Sanuki, T., Sasaki, M., Seo, E.S., Streitmatter, R.E., Suzuki, J., Tanaka, K., Yamagami, T., Yamamoto, A., Yoshida, T. and Yoshimura, K. (2007) Measurements of 0.2-20 GeV/n Cosmic-Ray Proton and Helium Spectra from 1997 through 2002 with the BESS Spectrometer. Astroparticle Physics, 28, 154-167. https://doi.org/10.1016/j.astropartphys.2007.05.001

[51] Cooper, J.A. (1970) Factors Determining the Ultimate Detection Sensitivity of $\mathrm{Ge}(\mathrm{Li})$ Gamma-Ray Spectrometers. Nuclear Instruments and Methods, 82, 273-277. https://doi.org/10.1016/0029-554X(70)90361-7

[52] Knoll, G.F. (2010) Radiation Detection and Measurement. 4th Edition, John Wiley \& Sons, Inc., Hoboken.

[53] Owens, A. and Peacock, A. (2004) Compound Semiconductor Radiation Detectors. Nuclear Instruments and Methods A, 531, 18-37. https://doi.org/10.1016/j.nima.2004.05.071

[54] Owens, A. (2008) Scintillators on Interplanetary Space Missions. IEEE Transactions on Nuclear Science, 55, 1430-1436. https://doi.org/10.1109/TNS.2008.921936

[55] Kozyrev, A., Mitrofanov, I., Owens, A., Quarati, F., Benkho, J., Bakhtin, B., Fedosov, F., Golovin, D., Litvak, M., Malakhov, A., Mokrousov, M., Nuzhdin, I., Sanin, A., Tretyakov, V., Vostrukhin, A., Timoshenko, G., Shvetsov, V., Granja, C., Slavicek, T. and Pospisil, S. (2016) A Comparative Study of $\mathrm{LaBr} 3(\mathrm{Ce} 3+)$ and $\mathrm{CeBr} 3$ Based Gamma-Ray Spectrometers for Planetary Remote Sensing Applications. Review of Scientific Instruments, 87, 85112. https://doi.org/10.1063/1.4958897

[56] Kobayashi, M., Hasebe, N., Miyachi, T., Fujii, M., Shibamura, E., Okudaira, O., Karouji, Y., Hareyama, M., Takashima, T., Kobayashi, S., D’Uston, C., Maurice, S., Yamashita, N. and Reedy, R.C. (2013) The Kaguya Gamma-Ray Spectrometer: Instrumentation and In-Flight Performances. Journal of Instrumentation, 8, 04010. https://doi.org/10.1088/1748-0221/8/04/P04010

[57] Evans, L.G., Peplowski, P.N., Rhodes, E.A., Lawrence, D.J., McCoy, T.J., Nittler, L.R., Solomon, S.C., Sprague, A.L., Stockstill-Cahill, K.R., Starr, R.D., Weider, S.Z., Boynton, W.V., Hamara, D.K. and Goldsten, J.O. (2012) Major-Element Abundances on the Surface of Mercury: Results from the MESSENGER Gamma-Ray 
Spectrometer. Journal of Geophysical Research, 117, E00L07. https://doi.org/10.1029/2012JE004178

[58] McCutchan, E.A. and Johnson, T. (2016) Evaluated Nuclear Structure Data File (ENSDF). http://www.nndc.bnl.gov/ensdf/

[59] Camp, A., Vargas, A. and Fernandez-Varea, J.M. (2016) Determination of LaBr3(Ce) Internal Background Using a HPGe Detector and Monte Carlo Simulations. Applied Radiation and Isotopes, 109, 512-517.

https://doi.org/10.1016/j.apradiso.2015.11.093

[60] Quarati, F.G.A., Khodyuk, I.V., van Eijk, C.W.E., Quarati, P. and Dorenbos, P. (2012) Study of 138La Radioactive Decays Using LaBr3 Scintillators. Nuclear Instruments and Methods A, 683, 46-52. https://doi.org/10.1016/j.nima.2012.04.066

[61] Combes, C.M., Dorenbos, P., van Eijk, C.W.E., Krämer, K.W. and Güdel, H.U. (1999) Optical and Scintillation Properties of Pure and Ce3+-Doped $\mathrm{Cs}_{2} \mathrm{LiYCl}_{6}$ and $\mathrm{Li}_{3} \mathrm{YCl}_{6}: \mathrm{Ce} 3+$ Crystals. Journal of Luminescence, 82, 299-305. https://doi.org/10.1016/S0022-2313(99)00047-2

[62] D’Olympia, N., Chowdhury, P., Guess, C.J., Harrington, T., Jackson, E.G., Lakshmi, S., Lister, C.J., Glodo, J., Hawrami, R., Shah, K. and Shirwadkar, U. (2012) Optimizing $\mathrm{Cs}_{2} \mathrm{LiYCl}_{6}$ for Fast Neutron Spectroscopy. Nuclear Instruments and $\mathrm{Me}$ thods A, 694, 140-146. https://doi.org/10.1016/j.nima.2012.07.021

[63] Glodo, J., Hawrami, R. and Shah, K.S. (2013) Development of $\mathrm{Cs}_{2} \mathrm{LiYCl}_{6}$ Scintillator. Journal of Crystal Growth, 379, 73-78. https://doi.org/10.1016/j.jcrysgro.2013.03.023

[64] Smith, M.B., Achtzehn, T., Andrews, H.R., Clifford, E.T.H., Forget, P., Glodo, J., Hawrami, R., Ing, H., O’Dougherty, P., Shah, K.S., Shirwadkar, U., Soundara-Pandian, L. and Tower, J. (2015) Fast Neutron Measurements Using $\mathrm{Cs}_{2} \mathrm{LiYCl}_{6}: \mathrm{Ce}$ (CLYC) Scintillator. Nuclear Instruments and Methods A, 784, 162-167. https://doi.org/10.1016/j.nima.2014.09.021

[65] Evans, L.G., Reedy, R.C., Starr, R.D., Kerry, K.E. and Boynton, W.V. (2006) Analysis of Gamma Ray Spectra Measured by Mars Odyssey. Journal of Geophysical Research, 112, 3-4.

[66] Naito, M., Hasebe, N., Yoshida, K., Ishii, J., Aoki, D., Nagaoka, H., Kusano, H. and Shibamura, E. (2016) Neutron Fluxes from Martian Satellites as a Function of Chemical Composition and Hydrogen Content. JPS Conference Proceedings, 11, Article ID: 050003. https://doi.org/10.7566/JPSCP.11.050003 